\title{
Thermodynamic Analysis of Azeotropic mixtures for Vapour liquid Equilibrium Determination-Experimental and Theoretical studies
}

\author{
Manojkumar Selvakumar and Sivaprakash Baskaran \\ Department of Chemical Engineering, FEAT, Annamalai University, Annamalai Nagar- 608002, India.
}

Correspondence Author: Manojumar Selvaumar, Annamalai University Department of Chemical Engineering Faculty of Engineering and Technology Annamalai Nagar, India - 608002

Received date: 2 December 2017, Accepted date: 22 January 2018, Online date: 28 February 2018

Copyright: (c 2018 Manojkumar Selvakumar. This is an open-access article distributed under the terms of the Creative Commons Attribution License, which permits unrestricted use, distribution, and reproduction in any medium, provided the original author and source are credited.

\begin{abstract}
Background and objective: Knowledge of precise vapor-liquid equilibria is a requirement to the detailed design of distillation equipment. However, such data is limited, and usually not available when new systems are under consideration, because it is complex and laborious to obtain the data experimentally. Predictive methods are therefore valuable for process evaluation and design. In this paper five binary azeotropes namely Acetone-water, Acetone-methanol, Ethanol-water, Ethanol-benzene, and Methanol-water were taken. Methodology: The experimental vapour liquid equilibrium data determination for this system was carried out using Othmer type ebuilliometer in laboratory scale at atmospheric pressure. For the theoretical prediction of VLE five activity coefficient models namely NRTL, UNIQUAC, UNIFAC and modified form of florry-huggins equations (SRS and TCRS) have been employed. The parameters for the five systems of four models namely NRTL, UNIQUAC SRS and TCRS were computed using Newton Raphson technique. UNIFAC model was adopted using Analytical solution of group contribution (ASOG) method. Also these models are validated using thermodynamic consistency test. Result: The result shown that all five azeotropic systems yield positive deviation from ideality due to dissimilar molecular structures. Conclusion: Of the chosen five activity coefficient models TCRS model give better result for Acetonewater System. UNIFAC model provide good representation of VLE for Acetone-methanol and Methanol-water system. Whereas NRTL and SRS models good agreement with Ethanol-water and Ethanol-benzene systems.
\end{abstract}

Key words: Vapour liquid Equilibrium, Azeotrope, Non ideal system, Activity Coefficient model, Thermodynamic consistency

\section{INTRODUCTION}

Separation of chemical in to the constituents is an art for millennia (Seader, J.D., J. Henley, 2006). Chemical Engineers are more concerned with separations process. Separation methods include distillation, absorption, liquid-liquid extraction, leaching, drying and crystallization etc (Geankoplis, C.J., 2003). Distillation, which is the most widely, used separation technique in the chemical process industries (Vivek Julka, et al., 2019) accounts for about 3\% of the world energy consumption. Also, it has substantial advantages over the other processes applied in order to separate a mixture, such extraction, crystallization, semi permeable membranes etc. Distillation process is based on the fact that the composition of the boiling liquid and that of the vapour over it differ. Thus, if the boiling temperature is low (e.g., air separation), it is necessary to use low temperature refrigerants and conduct the process at a higher pressure. If it is high (e.g., in separation of heavy oil fractions or metals), high temperature heat carries or fire preheating have to be used and the process is run under vacuum (Mohamad Azamudin, I., 2010). Because of the high energy demand of these processes the optimal design and operation of the distillation equipments are important from economic and also environmental points of view (Laszlo, H., 2013). The presence of azeotropic mixture however complicates the design of ordinary distillation principles (Gadekar, S.V., et al., 2004). It is impossible to conduct the distillation process in the case of azeotropic composition. Azeotropes or close-boiling mixtures (Narayanan, K.V., 2004). The molecular interactions when two or more components are mixed may cause the mixture to form certain "inseparable" compositions where the vapor and liquid compositions at equilibrium are equal within a given pressure and temperature range. These specific mixture compositions are called azeotropes. The defining condition of an azeotropic mixture and the physical phenomena leads to nonideality. Nonideal mixtures exhibit positive $\left(\gamma_{\mathrm{i}}>1\right)$ or negative $\left(\gamma_{\mathrm{i}}<1\right)$ deviations from Raoult's law (Hilmen, E.K., 2000). If these deviations become so large that the vapor pressure exhibits an extremal point at constant temperature, or, equivalently, an extremal point in the boiling temperature at constant pressure (Swietoslawski, W., 1963). Azeotropes play an important role in vapor-liquid equilibrium separation processes. For efficient design of distillation equipment or any other separation processes which are diffusional in nature requires quantitative understanding of vapour liquid equilibria. In Vapour liquid equilibrium phases are expressed through vapour phase fugacity coefficients and the liquid phase activity coefficients. At low or modest pressures fugacity coefficient can be estimated easily for very simple mixtures or ideal solutions, but for non-ideal mixtures, estimation of liquid phase activity coefficient is quite difficult (Managobinda, B., 2010). In the present work five azeotropic systems namely acetone-methanol, chloroform-methanol, acetone-water, ethanol-benzene and methanol-water were taken for study. Experimental VLE of these systems were determined using an Othmer VLE still. Applicability of five activity coefficient models to these systems were tested in the study viz. NRTL, 
UNIQUAC, UNIFAC and two forms of modified Flory - Huggins equations (SRS and TCRS). Also, thermodynamic consistency test for these models was carried out by Redlich Kister method.

2.1 Experimentation:

\section{MATERIALS AND METHODS}

All the chemicals (acetone, benzene, ethanol, and methanol) were supplied by the Indian Scientific Chemical Industries Pvt. Ltd., Chennai, India. The purities of all reagents are confirmed to be analytical grade by gas chromatography and were found to be 0.998 mass fractions for ethanol, methanol 0.997 mass fractions for acetone, benzene. The water used in the experiment is deionised water which is prepared in the laboratory.

\subsubsection{Experimental Procedure:}

Othmer VLE still (see fig.1) was employed to determine vapour liquid equilibrium data. The capacity of the still is about $100 \mathrm{ml}$ and it is outfitted with reflux condenser. Binary liquid mixture of known composition was charged at the top of the VLE still and distilled using electrical heating. The distillate (vapour form) richer in more volatile compound enters the condenser with cold water circulation and is collected at the top. The residual product (liquid) richer in less volatile compound can be collected from the bottom. The still is equipped with a quartz thermometer to measure the azeotropic distillation temperature. After equilibrium was established (indicated by a constant reading in the thermometer), heating was stopped and the contents of the top and bottom products were allowed to cool and analyzed. The samples were analyzed using Clarus $680 \mathrm{GC}$ fused with silica column and packed with Elite-5MS (5\% biphenyl 95\% dimethylpolysiloxane, 30 $\mathrm{m} \times 0.25 \mathrm{~mm} \mathrm{ID} \times 250 \mu \mathrm{m} \mathrm{df}$ ). The components were separated using Helium as carrier gas at a constant flow of $1 \mathrm{ml} / \mathrm{min}$. The injector temperature was set at $260^{\circ} \mathrm{C}$ during the chromatographic run. One $\mu \mathrm{L}$ of extract sample was injected into the instrument and the oven temperature was at $60^{\circ} \mathrm{C}(2 \mathrm{~min})$, followed by $300^{\circ} \mathrm{C}$ at the rate of $10^{\circ} \mathrm{C} \mathrm{min}-1$ and $300^{\circ} \mathrm{C}$, where it was held for $6 \mathrm{~min}$. The mass detector conditions were: transfer line temperature $240^{\circ} \mathrm{C}$, ion source temperature $240^{\circ} \mathrm{C}$, and ionization mode electron impact at 70 electonvolt, a scan time 0.2 seconds and scan interval of 0.1 seconds. The spectra of the components were compared with the database of spectra of known components stored in the GC-MS NIST (2008) library.

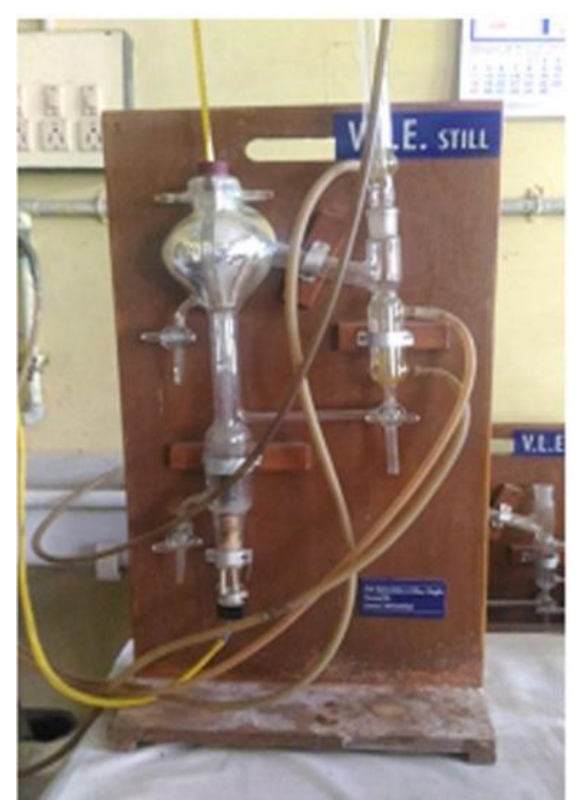

Fig. 1: Othmer VLE Still

2.1.3 Low pressure VLE data reduction:

There are different methods available for the regression of isothermal and isobaric VLE data. The gamma/phi $(\gamma-\varphi)$ formulation of VLE or more commonly known as the combined method was used in this work to regress the VLE data (Ngema, 2010). The combined method uses an equation of state to calculate the fugacity coefficients that describe the vapour phase non-idealities, while an activity coefficient model is used to calculate the activity coefficients that describe the liquid phase non-idealities. The gamma/phi method relies upon liquid phase activity coefficient models to represent VLE data, and give accurate result for non ideal solution (Kannan, A., 2003) which is given by

$\varphi_{\mathrm{i}} \mathrm{y}_{\mathrm{i}} \mathrm{P}=\gamma_{\mathrm{i}} \mathrm{x}_{\mathrm{i}} \mathrm{P}_{\mathrm{i}}^{\text {Sat }}$

$\mathrm{y}_{\mathrm{i}}$ is mole fraction in vapour phase; $\mathrm{x}_{\mathrm{i}}$ is mole fraction in liquid phase; $\mathrm{P}_{\mathrm{i}}^{\mathrm{sat}}$ is vapour pressure and $\mathrm{P}$ is operating pressure; $\varphi_{i}$ is fugacity coefficient and $\gamma_{i}$ is activity coefficient.

2.1.4 Fugacity Coefficient:

At low to moderate pressure (0 to $10 \mathrm{~atm}$ ), fugacity coefficient can be calculated by the following equations (Rao, Y.V.C., 1997).

$\ln \varphi_{\mathrm{i}}=\left(\mathrm{B}^{0}+\omega \mathrm{B}^{1}\right) \mathrm{P}_{\mathrm{r}} / \mathrm{T}_{\mathrm{r}}$

$\mathrm{f}_{\mathrm{i}}=\varphi_{\mathrm{i}} \mathrm{P}$

$\mathrm{B}_{0}=0.083-0.422 / \mathrm{T}_{\mathrm{r}}{ }^{1.6}$

$\mathrm{B}^{1}=0.139-0.172 / \mathrm{T}_{\mathrm{r}}^{4.2}$

$\phi_{\mathrm{i}}$ is fugacity coefficient; $\mathrm{B}^{0} \& \mathrm{~B}^{1}$ are virial coefficients; $\omega$ is accentric factor; $\mathrm{P}_{\mathrm{r}}$ is reduced pressure; $\mathrm{T}_{\mathrm{r}}$ is reduced temperature. 
2.5.1 NRTL (Non-Random Two Liquid) Model:

The non random two liquid (NRTL) equation proposed by Renon (1968) is applicable to partially miscible as well as completely miscible systems. The equations for the activity coefficients are

$\ln \gamma_{1}=x_{2}^{2}\left[\tau_{21}\left(\frac{G_{21}}{x_{1}+x_{2} G_{21}}\right)^{2}+\frac{\tau_{12} G_{12}}{\left(x_{2}+x_{1} G_{12}\right)^{2}}\right]$
$\ln \gamma_{2}=x_{1}^{2}\left[\tau_{12}\left(\frac{G_{12}}{x_{2}+x_{1} G_{12}}\right)^{2}+\frac{\tau_{21} G_{21}}{\left(x_{1}+x_{2} G_{21}\right)^{2}}\right]$

where $\mathrm{G}_{12}$ and $\mathrm{G}_{21}$ energy interaction between the molecules

2.5.2 UNIQUAC (UNIversal Quasi-Chemical) model:

The UNIQUAC equation was developed by Abrams and Prausnitz (1978) who incorporated the two-liquid model and the theory of local composition. The UNIQUAC equation consists of two parts a combinatorial part that takes into accounts the differences in sizes and shapes of the molecules and the residual part that is due to the intermolecular forces between the molecules. In the form of an equation, this is represented as

$$
\begin{aligned}
& \ln \gamma_{\mathrm{i}}^{\mathrm{C}}=\ln \frac{\phi_{\mathrm{i}}}{\mathrm{x}_{\mathrm{i}}}+\frac{z}{2} \mathrm{q}_{\mathrm{i}} \ln \frac{\theta_{\mathrm{i}}}{\phi_{\mathrm{i}}}+\mathrm{l}_{\mathrm{i}}-\frac{\phi_{i}}{\mathrm{x}_{\mathrm{i}}} \Sigma_{\mathrm{j}} \mathrm{x}_{\mathrm{j}}{ }_{\mathrm{j}} \\
& \ln \gamma_{\mathrm{i}}^{\mathrm{R}}=\mathrm{q}_{\mathrm{i}}\left[1-\ln \left(\Sigma_{\mathrm{j}} \theta_{\mathrm{j}} \tau_{\mathrm{ji}}\right)-\Sigma_{\mathrm{j}} \frac{\theta_{\mathrm{j}} \tau_{\mathrm{ij}}}{\Sigma_{\mathrm{k}} \theta_{\mathrm{k}} \tau_{\mathrm{kj}}}\right.
\end{aligned}
$$

$\mathrm{l}_{\mathrm{i}}=\frac{\mathrm{z}}{2}\left(\mathrm{r}_{\mathrm{i}}-\mathrm{q}_{\mathrm{i}}\right)-\left(\mathrm{r}_{\mathrm{i}}-1\right)$

$\mathrm{r}_{\mathrm{i}}=\Sigma_{\mathrm{k}} \mathrm{v}_{\mathrm{k}}^{\mathrm{i}} \mathrm{R}_{\mathrm{k}}$

$\mathrm{q}_{\mathrm{i}}=\Sigma_{\mathrm{k}} \mathrm{v}_{\mathrm{k}}^{\mathrm{i}} \mathrm{Q}_{\mathrm{k}}$

where $\phi_{i}=$ segment or volume fraction of the component

$\theta \mathrm{i}=$ area fraction of the component

$r_{i}=$ volume parameter of the component

$\mathrm{q}_{\mathrm{i}}=$ surface area parameter of the component

The UNIQUAC equation contains only two adjustable parameters $\tau_{12}$ and $\tau_{12}$.

2.5.3 UNIquac Functional group Activity Coefficient (UNIFAC) method:

UNIFAC is based on UNIQUAC model, has a combinatorial term that depends on the volume and surface area of each molecule and a residual term that is the result of the energies of interaction between the molecules (Fredenslund, A., et al., 1977). The combinatorial term is evaluated using equation (8) When using the UNIFAC model one first identifies the functional subgroups present in each molecule. Next the activity coefficient for each species is written as (Fredenslund, A., et al., 1975)

$\ln \gamma_{\mathrm{i}}=\ln \gamma_{\mathrm{i}}$ (combinatorial) $+\ln \gamma_{\mathrm{i}}$ (residual)

$\ln \gamma_{i}($ residual $)=\Sigma_{\mathrm{k}} \mathrm{v}_{\mathrm{k}}^{\mathrm{i}}\left[\ln \Gamma_{\mathrm{k}}-\ln \Gamma_{\mathrm{k}}^{\mathrm{i}}\right]$

$\ln \Gamma_{\mathrm{K}}=\mathrm{Q}_{\mathrm{k}}\left[1-\ln \left(\Sigma_{\mathrm{m}} \varphi_{\mathrm{m}} \Psi_{\mathrm{mk}}\right)-\Sigma_{\mathrm{m}} \frac{\varphi_{\mathrm{m}}-\Psi_{\mathrm{km}}}{\Sigma_{\mathrm{n}} \varphi_{\mathrm{n}} \Psi_{\mathrm{nm}}}\right]$

$\Psi_{\mathrm{mn}}=\exp -\left[\frac{\mathrm{u}_{\mathrm{mn}}-\mathrm{u}_{\mathrm{nn}}}{\mathrm{RT}}\right]=\exp \frac{-\mathrm{a}_{\mathrm{mn}}}{\mathrm{T}}$

where $\Gamma_{\mathrm{k}}^{\mathrm{i}}$ is residual activity coefficient; $\mathrm{a}_{\mathrm{mn}}$ is interaction parameter; $\mathrm{u}_{\mathrm{mn}}$ is interaction energy between group $\mathrm{m}$ and $\mathrm{n}$.

2.6 Modified form of Flory - Huggins equation:

2.6.1 Simplified Ruckenstein and Shulgin model (SRS):

Ruckenstein and Shulgin modified the local composition and Flory-Huggins equations (F-H) for non electrolyte solutions. Their equations for ln $\gamma_{i}$ are (Sabarathinam, P.L., B. Sivaprakash, 2002)

$\ln \gamma_{1}=-\ln \left(\mathrm{x}_{1}+\mathrm{x}_{2} \mathrm{~L}_{12}\right)-\mathrm{x}_{2}\left[\mathrm{~A}_{12}-\mathrm{A}_{21}\right]-\left(\ln \mathrm{L}_{12}+\ln \mathrm{L}_{21}\right)\left[\varphi_{1} \varphi_{2}+\mathrm{x}_{2} \varphi_{2} \frac{\partial \varphi_{1}}{\partial \mathrm{x}_{1}}+\mathrm{x}_{2} \varphi_{1} \frac{\partial \varphi_{2}}{\partial \mathrm{x}_{1}}\right]$ 
$\ln \gamma_{2}=-\ln \left(\mathrm{x}_{2}+\mathrm{x}_{1} \mathrm{~L}_{21}\right)-\mathrm{x}_{1}\left[\mathrm{~A}_{12}-\mathrm{A}_{21}\right]-\left(\ln \mathrm{L}_{12}+\ln \mathrm{L}_{21}\right)\left[\varphi_{1} \varphi_{2}+\mathrm{x}_{1} \varphi_{2} \frac{\partial \varphi_{1}}{\partial \mathrm{x}_{1}}+\mathrm{x}_{1} \varphi_{1} \frac{\partial \varphi_{2}}{\partial \mathrm{x}_{1}}\right]$

Where $A_{i j}=\left[\frac{L_{i j}}{x_{1}+x_{2} L_{i j}}\right]$

$\mathrm{L}_{\mathrm{ij}}=\frac{\mathrm{V}_{\mathrm{j}}}{\mathrm{V}_{\mathrm{i}}} \exp \left[-\frac{\lambda_{\mathrm{ij}-} \lambda_{\mathrm{ii}}}{\mathrm{RT}}\right]$

$\varphi_{\mathrm{i}}=\frac{\mathrm{x}_{1}}{\mathrm{x}_{1}+\mathrm{x}_{2} \mathrm{~L}_{\mathrm{ji}}}$

$\varphi_{\mathrm{j}}=\frac{\mathrm{x}_{2}}{\mathrm{x}_{2}+\mathrm{x}_{1} \mathrm{~L}_{\mathrm{ji}}}$

where $\mathrm{x}_{1}$ and $\mathrm{x}_{2}$ are mole fraction in liquid phase. $\mathrm{A}_{12}$ and $\mathrm{A}_{21}$ are two adjustable parameters related to pure component molar volume and characteristic energy difference. $\varphi_{1}$ and $\varphi_{2}$ are segment fraction of the components and $\chi$ is an energy interaction between molecules of components.

2.6.2 Theoretically Consistent Ruckenstein and Shulgin model (TCRS):

Like NRTL equation the new equation (TCRS) is also three parameter models. Their expression for $\ln \gamma_{\mathrm{i}}$ 's are (Sabarathinam, P.L., B. Sivaprakash, 2002)

$\ln \gamma_{1}=-\ln \left(x_{1}+x_{2} L_{12} \exp \left(x_{1} \delta_{12}\right)\right)+x_{2}\left[A_{i j}-A_{j i}\right]-x_{1} x_{2}{ }^{2} \delta_{12}\left(A_{i j}-A_{j i}\right)-\left(\ln L_{12}+\ln L_{21}+\delta_{12}\left(x_{1}-x_{2}\right)\right)$

$\left[\varphi_{1} \varphi_{2}+x_{2} \varphi_{2} \frac{\partial \varphi_{1}}{\partial x_{1}}+x_{2} \varphi \frac{\partial \varphi_{2}}{\partial x_{1}}\right]-2 x_{2} \varphi_{1} \varphi_{2} \delta_{12}$

$\ln \gamma_{2}=-\ln \left(\mathrm{x}_{2}+\mathrm{x}_{1} \mathrm{~L}_{21} \exp \left(-\mathrm{x}_{2} \delta_{12}\right)\right)-\mathrm{x}_{1}\left[\mathrm{~A}_{12}-\mathrm{A}_{21}\right]-\mathrm{x}_{1}{ }^{2} \mathrm{x}_{2} \delta_{12}\left(\mathrm{~A}_{12}-\mathrm{A}_{21}\right)-\left(\operatorname{lnL}{ }_{12}+\operatorname{lnL}{ }_{21}+\delta_{12}\left(\mathrm{x}_{1}-\mathrm{x}_{2}\right)\right)$

$\left[\varphi_{1} \varphi_{2}-x_{1} \varphi_{2} \frac{\partial \varphi_{1}}{\partial x_{1}}+x_{1} \varphi_{1}-x_{1} \varphi_{1} \frac{\partial \varphi_{2}}{\partial x_{1}}\right]+2 x_{1} \varphi_{1} \varphi_{2} \delta_{12}$

where $\quad \mathrm{Lij}=\frac{V_{j}}{V_{i}} \exp \left[\frac{\lambda_{i j}-\lambda_{i i}}{R T}\right]$

$\delta_{12}=\left[\frac{\lambda_{12}-\lambda_{21}}{R T}\right]$

$\mathrm{A}_{\mathrm{ij}}=\left[\frac{L_{i j} \exp \left(x_{1} \delta_{12}\right)}{x_{1}+x_{2} L_{i j} \exp \left(x_{1} \delta_{12}\right)}\right]$

$\varphi_{i}=\frac{x_{1}}{x_{1}+x_{2} L_{i j} \exp \left[x_{1} \delta_{12}\right]}$

$\varphi_{j}=\frac{x_{2}}{x_{2}+x_{1} L_{j i} \exp \left[-x_{2} \delta_{12}\right]}$

2.7 Error Analysis:

The relative error percentages of the activity coefficient models are calculated using equation

$\mathrm{REy}_{1}=\frac{\mid \mathrm{y}_{1} \text { Experiment al - } \mathrm{y}_{1} \text { Calculated } \mid}{\mathrm{y}_{1} \text { Experimental }} \times 100$

2.8 VLE Consistency:

The Gibbs duhem equation relates the chemical potential of the components in a mixture to another. When applied to liquid phase and written in the terms of activity coefficients, the isothermal and isobaric form for a binary system is (Philip Jackson, L., A. Richard Wilsak, 1995)

$\mathrm{X}_{1} \mathrm{~d} \ln \gamma_{1}+\mathrm{X}_{2} \mathrm{~d} \ln \gamma_{2}=0$

This total differential can be manipulated into various forms each giving rise to different thermodynamic consistency test. Of all the thermodynamic consistency tests the integral test is probably the best known. It was proposed by RedlichKister in 1948 (Redlich, O., A.T. Kister, 1948). In this work the thermodynamic consistency of measured (vapour + liquid) equilibrium data is validated using integral test it is given by 
$\int_{0}^{1} \ln \frac{\gamma_{1}}{\gamma_{2}} \mathrm{dx}_{1}=0$

The limits of integrations cover the entire composition range. The integral test can be applied rigorously to isothermal and isobaric activity coefficient. The graphical implication is straight forward. If $\ln \gamma_{1} / \gamma_{2}$ is plotted against $x_{1}$, the area above the axis should equal to area below it.

\section{RESULT AND DISCUSSION}

VLE data for the five binary systems namely Acetone-water, Acetone-methanol, Ethanol-water, Ethanol-benzene, and Methanol-water were determined and the results are represented in tables 1-5. The experimental data are compared with literature and found to be in good agreement. Vapour phase ideality was characterized by fugacity coefficient calculation using equations (2-5) from the calculations the fugacity coefficient was found to be closer to unity. Hence it is reasonable to consider that the vapour phase is ideal. The activity coefficient $\left(\gamma_{1}\right)$ calculated from the experimental VLE using Equation (1) for the five azeotropic systems is also incorporated in tables $1-5$. Since the numerical values of activity coefficient $\left(\gamma_{1}\right)$ are greater than unity for all the systems the liquid phase feature strong non ideality. This is due to the fact that the liquid phase molecules are much closely spaced than in vapor phase due to which attraction / repulsion among the molecules are high (Luben, W.L., L.A. Wenzel, 1988). Also all the five azeotropic systems show positive deviation from ideality (minimum boiling azeotropes). This is because the dissimilar molecular structures of five azeotropic systems exert repulsive forces other than attractive forces. The repulsive forces results in higher concentration of molecules in vapour phase than in the liquid phase with higher activity coefficient $(\gamma)$.

Table 1: Experimental and Models Predicted VLE of Acetone-water System at $101.325 \mathrm{kPa}$

\begin{tabular}{|c|c|c|c|c|c|c|c|c|c|c|c|c|c|c|c|c|c|c|}
\hline \multirow[b]{2}{*}{$\mathrm{T}$} & \multirow[b]{2}{*}{$\mathrm{X}$} & \multirow[b]{2}{*}{ Y } & \multirow[b]{2}{*}{$\begin{array}{l}\gamma_{1} \\
\text { Exp } \\
\end{array}$} & \multicolumn{3}{|c|}{ NRTL } & \multicolumn{3}{|c|}{ UNIQUAC } & \multicolumn{3}{|c|}{ UNIFAC } & \multicolumn{3}{|c|}{ SRS } & \multicolumn{3}{|c|}{ TCRS } \\
\hline & & & & $\gamma_{1}$ & $\mathrm{y}_{1}$ & $\begin{array}{l}\mathrm{y}_{1} \\
\text { Error } \\
\%\end{array}$ & $\gamma_{1}$ & $\mathrm{y}_{1}$ & $\begin{array}{l}\mathrm{y}_{1} \\
\text { Error } \\
\%\end{array}$ & $\gamma_{1}$ & $\mathrm{y}_{1}$ & $\begin{array}{ll}y_{1} & \text { Error } \\
\% & \end{array}$ & $\gamma_{1}$ & $\mathrm{y}_{1}$ & $\begin{array}{l}\mathrm{y}_{1} \\
\text { Error } \\
\%\end{array}$ & $\gamma_{1}$ & $\mathrm{y}_{1}$ & $\begin{array}{l}\mathrm{y}_{1} \\
\text { Erro } \\
\mathrm{r} \%\end{array}$ \\
\hline 74. & 0.06 & 0.59 & 5.33 & 7.15 & 0.80 & 46.27 & 3.64 & 0.40 & 31.70 & 6.41 & 0.71 & 20.2976 & 2. & 0.33 & 44.05 & 4.89 & 0.54 & 8.25 \\
\hline 80 & 15 & 81 & 29 & 65 & 26 & 30 & 31 & 85 & 03 & 57 & 95 & & $\begin{array}{l}98 \\
35\end{array}$ & 46 & 61 & 27 & 87 & 94 \\
\hline 68. & 0.12 & 0.74 & 3.92 & 4.60 & 0.86 & 12.29 & 3.46 & 0.65 & 11.70 & 4.38 & 0.82 & 11.6741 & 3. & 0.68 & 7.674 & 4.10 & 0.77 & 4.51 \\
\hline 53 & 53 & 01 & 65 & 83 & 86 & 47 & 71 & 35 & 11 & 50 & 65 & & $\begin{array}{l}62 \\
54\end{array}$ & 33 & & 39 & 35 & 29 \\
\hline $\begin{array}{l}65 . \\
26\end{array}$ & $\begin{array}{l}0.14 \\
92\end{array}$ & $\begin{array}{l}0.76 \\
16\end{array}$ & $\begin{array}{l}3.76 \\
36\end{array}$ & $\begin{array}{l}4.28 \\
06\end{array}$ & $\begin{array}{l}0.86 \\
62\end{array}$ & $\begin{array}{l}8.737 \\
1\end{array}$ & $\begin{array}{l}3.42 \\
17\end{array}$ & $\begin{array}{l}0.69 \\
24\end{array}$ & $\begin{array}{l}9.086 \\
1\end{array}$ & $\begin{array}{l}4.10 \\
22\end{array}$ & $\begin{array}{l}0.83 \\
01\end{array}$ & 8.9942 & $\begin{array}{l}3 . \\
93\end{array}$ & $\begin{array}{l}0.79 \\
59\end{array}$ & $\begin{array}{l}4.503 \\
6\end{array}$ & $\begin{array}{l}3.93 \\
68\end{array}$ & $\begin{array}{l}0.79 \\
66\end{array}$ & 4.59 \\
\hline & & & & & & & & & & & & & 32 & & & & & \\
\hline $\begin{array}{l}63 . \\
59\end{array}$ & $\begin{array}{l}0.21 \\
24\end{array}$ & $\begin{array}{l}0.79 \\
12\end{array}$ & $\begin{array}{l}2.89 \\
86\end{array}$ & $\begin{array}{l}2.70 \\
45\end{array}$ & $\begin{array}{l}0.85 \\
58\end{array}$ & $\begin{array}{l}2.589 \\
3\end{array}$ & $\begin{array}{l}2.70 \\
45\end{array}$ & $\begin{array}{l}0.73 \\
82\end{array}$ & $\begin{array}{l}6.698 \\
6\end{array}$ & $\begin{array}{l}3.04 \\
37\end{array}$ & $\begin{array}{l}0.83 \\
08\end{array}$ & 5.005 & $\begin{array}{l}3 . \\
20\end{array}$ & $\begin{array}{l}0.87 \\
58\end{array}$ & $\begin{array}{l}10.69 \\
2\end{array}$ & $\begin{array}{l}3.05 \\
63\end{array}$ & $\begin{array}{l}0.83 \\
42\end{array}$ & $\begin{array}{l}5.43 \\
47\end{array}$ \\
\hline $\begin{array}{l}60 . \\
75\end{array}$ & $\begin{array}{l}0.32 \\
14\end{array}$ & $\begin{array}{l}0.82 \\
04\end{array}$ & $\begin{array}{l}2.17 \\
97\end{array}$ & $\begin{array}{l}2.19 \\
42\end{array}$ & $\begin{array}{l}0.82 \\
58\end{array}$ & $\begin{array}{l}0.398 \\
0\end{array}$ & $\begin{array}{l}2.03 \\
18\end{array}$ & $\begin{array}{l}0.76 \\
47\end{array}$ & $\begin{array}{l}6.789 \\
3\end{array}$ & $\begin{array}{l}2.19 \\
18\end{array}$ & $\begin{array}{l}0.82 \\
49\end{array}$ & 0.5485 & $\begin{array}{l}2 . \\
35 \\
3\end{array}$ & $\begin{array}{l}0.88 \\
56\end{array}$ & $\begin{array}{l}7.947 \\
3\end{array}$ & $\begin{array}{l}2.20 \\
29\end{array}$ & $\begin{array}{l}0.82 \\
91\end{array}$ & $\begin{array}{l}1.06 \\
04\end{array}$ \\
\hline 59. & 0.42 & 0.83 & 1.74 & 1.65 & 0.79 & 2.274 & 1.62 & 0.77 & 7.217 & 1.70 & 0.81 & 2.6526 & 1. & 0.87 & 4.731 & 1.69 & 0.81 & 2.83 \\
\hline 95 & 01 & 69 & 68 & 88 & 47 & 9 & 09 & 65 & 1 & 07 & 47 & & $\begin{array}{l}82 \\
96\end{array}$ & 65 & 7 & 75 & 32 & 18 \\
\hline 59. & 0.49 & 0.83 & 1.52 & 1.40 & 0.77 & 4.340 & 1.42 & 0.78 & 6.557 & 1.47 & 0.80 & 3.4577 & 1. & 0.86 & 3.278 & 1.47 & 0.81 & 3.31 \\
\hline 12 & 61 & 87 & 40 & 96 & 57 & 8 & 41 & 37 & 7 & 14 & 97 & & $\begin{array}{l}57 \\
41\end{array}$ & 62 & 8 & 36 & 09 & 46 \\
\hline 58. & 0.61 & 0.85 & 1.30 & 1.18 & 0.78 & 3.535 & 1.21 & 0.80 & 6.284 & 1.22 & 0.81 & 5.412 & 1. & 0.84 & 1.955 & 1.23 & 0.81 & 5.20 \\
\hline 29 & 26 & 92 & 00 & 89 & 57 & 9 & 84 & 52 & 9 & 97 & 27 & & $\begin{array}{l}27 \\
47\end{array}$ & 24 & 3 & 25 & 45 & 25 \\
\hline 57. & 0.69 & 0.87 & 1.19 & 1.09 & 0.80 & 3.126 & 1.13 & 0.83 & 4.442 & 1.13 & 0.82 & 4.8668 & 1. & 0.82 & 5.084 & 1.13 & 0.82 & 4.90 \\
\hline 49 & 71 & 12 & 00 & 64 & 26 & 1 & 73 & 25 & 1 & 22 & 88 & & $\begin{array}{l}12 \\
96\end{array}$ & 69 & & 18 & 85 & 12 \\
\hline 56. & 0.78 & 0.88 & 1.11 & 1.04 & 0.83 & 2.798 & 1.09 & 0.87 & 1.776 & 1.07 & 0.85 & 3.4626 & 1. & 0.83 & 5.801 & 1.07 & 0.85 & 3.57 \\
\hline 68 & 38 & 95 & 07 & 11 & 37 & 1 & 11 & 37 & 2 & 24 & 87 & & $\begin{array}{l}04 \\
64\end{array}$ & 79 & 0 & 11 & 77 & 50 \\
\hline 56. & 0.89 & 0.92 & 1.02 & 1.00 & 0.90 & 1.014 & 1.03 & 0.93 & 0.796 & 1.01 & 0.91 & 0.6768 & 0. & 0.89 & 3.037 & 1.01 & 0.91 & 0.73 \\
\hline 30 & 23 & 33 & 60 & 82 & 73 & 6 & 42 & 07 & 0 & 91 & 71 & & $\begin{array}{l}99 \\
49\end{array}$ & 53 & 8 & 86 & 66 & 10 \\
\hline & & & & & & $\begin{array}{l}7.943 \\
8\end{array}$ & & & $\begin{array}{l}8.459 \\
0\end{array}$ & & & $\begin{array}{l}6.095 \\
2\end{array}$ & & & $\begin{array}{l}8.978 \\
5\end{array}$ & & & $\begin{array}{l}4.03 \\
80\end{array}$ \\
\hline
\end{tabular}

Table 2. Experimental and Models Predicted VLE of Acetone-methanol System at $101.325 \mathrm{kPa}$

\begin{tabular}{|c|c|c|c|c|c|c|c|c|c|c|c|c|c|c|c|c|c|c|}
\hline \multirow[b]{2}{*}{$\mathrm{T}$} & \multirow[b]{2}{*}{$X$} & \multirow[b]{2}{*}{$\mathrm{Y}$} & \multirow[b]{2}{*}{$\begin{array}{l}\gamma_{1} \\
\text { Exp }\end{array}$} & \multicolumn{3}{|c|}{ NRTL } & \multicolumn{3}{|c|}{ UNIQUAC } & \multicolumn{3}{|c|}{ UNIFAC } & \multicolumn{3}{|c|}{ SRS } & \multicolumn{3}{|c|}{ TCRS } \\
\hline & & & & $\gamma_{1}$ & $\mathrm{y}_{1}$ & $\begin{array}{l}\mathrm{y}_{1} \\
\text { Error } \\
\%\end{array}$ & $\gamma_{1}$ & $\mathrm{y}_{1}$ & $\begin{array}{l}\mathrm{y}_{1} \\
\text { Error } \\
\% \\
\end{array}$ & $\gamma_{1}$ & $\mathrm{y}_{1}$ & $\begin{array}{ll}\mathrm{y}_{1} & \text { Error } \\
\% & \end{array}$ & $\gamma_{1}$ & $\mathrm{y}_{1}$ & $\begin{array}{l}\mathrm{y}_{1} \\
\text { Error } \\
\%\end{array}$ & $\gamma_{1}$ & $\mathrm{y}_{1}$ & $\begin{array}{l}\mathrm{y}_{1} \\
\text { Error } \\
\%\end{array}$ \\
\hline $\begin{array}{l}65 . \\
5\end{array}$ & $\begin{array}{l}0.05 \\
61\end{array}$ & $\begin{array}{l}0.16 \\
73\end{array}$ & $\begin{array}{l}2.18 \\
59\end{array}$ & $\begin{array}{l}1.79 \\
31\end{array}$ & $\begin{array}{l}0.13 \\
72\end{array}$ & $\begin{array}{l}17.99 \\
16\end{array}$ & $\begin{array}{l}1.86 \\
59\end{array}$ & $\begin{array}{l}0.14 \\
28\end{array}$ & $\begin{array}{l}14.64 \\
43\end{array}$ & $\begin{array}{l}2.51 \\
71\end{array}$ & $\begin{array}{l}0.19 \\
26\end{array}$ & 15.1225 & $\begin{array}{l}2 . \\
96 \\
86\end{array}$ & $\begin{array}{l}0.22 \\
72\end{array}$ & $\begin{array}{l}35.80 \\
39 \\
\end{array}$ & $\begin{array}{l}1.62 \\
42\end{array}$ & $\begin{array}{l}0.12 \\
43\end{array}$ & $\begin{array}{l}25.70 \\
23\end{array}$ \\
\hline $\begin{array}{l}64 . \\
5\end{array}$ & $\begin{array}{l}0.11 \\
54\end{array}$ & $\begin{array}{l}0.29 \\
39\end{array}$ & $\begin{array}{l}1.92 \\
77\end{array}$ & $\begin{array}{l}1.76 \\
32\end{array}$ & $\begin{array}{l}0.26 \\
88\end{array}$ & $\begin{array}{l}8.540 \\
3\end{array}$ & $\begin{array}{l}1.78 \\
81\end{array}$ & $\begin{array}{l}0.27 \\
26\end{array}$ & $\begin{array}{l}7.247 \\
3\end{array}$ & $\begin{array}{l}1.97 \\
88\end{array}$ & $\begin{array}{l}0.30 \\
16\end{array}$ & 2.6199 & $\begin{array}{l}2 . \\
45 \\
84 \\
\end{array}$ & $\begin{array}{l}0.37 \\
48\end{array}$ & $\begin{array}{l}27.52 \\
63\end{array}$ & $\begin{array}{l}1.67 \\
52\end{array}$ & $\begin{array}{l}0.25 \\
54\end{array}$ & $\begin{array}{l}13.09 \\
96\end{array}$ \\
\hline $\begin{array}{l}63 . \\
5\end{array}$ & $\begin{array}{l}0.18 \\
85\end{array}$ & $\begin{array}{l}0.40 \\
94\end{array}$ & $\begin{array}{l}1.69 \\
79\end{array}$ & $\begin{array}{l}1.55 \\
46\end{array}$ & $\begin{array}{l}0.37 \\
48\end{array}$ & $\begin{array}{l}8.451 \\
3\end{array}$ & $\begin{array}{l}1.74 \\
85 \\
\end{array}$ & $\begin{array}{l}0.42 \\
15 \\
\end{array}$ & $\begin{array}{l}2.955 \\
5 \\
\end{array}$ & $\begin{array}{l}1.77 \\
24 \\
\end{array}$ & $\begin{array}{l}0.42 \\
73 \\
\end{array}$ & 4.3722 & $\begin{array}{l}1 . \\
98 \\
33 \\
\end{array}$ & $\begin{array}{l}0.47 \\
82 \\
\end{array}$ & $\begin{array}{l}16.80 \\
50\end{array}$ & $\begin{array}{l}1.79 \\
88\end{array}$ & $\begin{array}{l}0.43 \\
37 \\
\end{array}$ & $\begin{array}{l}5.935 \\
5 \\
\end{array}$ \\
\hline $\begin{array}{l}62 . \\
5\end{array}$ & $\begin{array}{l}0.31 \\
97\end{array}$ & $\begin{array}{l}0.52 \\
35\end{array}$ & $\begin{array}{l}1.32 \\
24\end{array}$ & $\begin{array}{l}1.25 \\
04\end{array}$ & $\begin{array}{l}0.49 \\
49\end{array}$ & $\begin{array}{l}5.463 \\
2\end{array}$ & $\begin{array}{l}1.39 \\
64\end{array}$ & $\begin{array}{l}0.55 \\
27\end{array}$ & $\begin{array}{l}5.577 \\
8 \\
\end{array}$ & $\begin{array}{l}1.38 \\
58\end{array}$ & $\begin{array}{l}0.54 \\
85\end{array}$ & 4.7755 & $\begin{array}{l}1 . \\
47 \\
21\end{array}$ & $\begin{array}{l}0.58 \\
27\end{array}$ & $\begin{array}{l}11.30 \\
85\end{array}$ & $\begin{array}{l}1.44 \\
94\end{array}$ & $\begin{array}{l}0.57 \\
37\end{array}$ & $\begin{array}{l}9.589 \\
3\end{array}$ \\
\hline 61. & 0.39 & 0.56 & 1.20 & 1.15 & 0.54 & 3.575 & 1.24 & 0.58 & 3.592 & 1.22 & 0.58 & 2.3423 & 1. & 0.61 & 8.453 & 1.36 & 0.64 & 13.34 \\
\hline
\end{tabular}


Citation: Manojkumar Selvakumar and Sivaprakash Baskaran, 2018. Advers Thermodynamic Analysis of Azeotropic mixtures for Vapour liquid Equilibrium Determination-Experimental and Theoretical studies. Journal of Applied Sciences Research., 14(1): 12-24.

\begin{tabular}{|c|c|c|c|c|c|c|c|c|c|c|c|c|c|c|c|c|c|c|}
\hline 5 & 45 & 78 & 11 & 84 & 75 & 2 & 44 & 82 & 8 & 94 & 11 & & $\begin{array}{l}30 \\
27\end{array}$ & 58 & 6 & 16 & 36 & 97 \\
\hline $\begin{array}{l}58 . \\
5\end{array}$ & $\begin{array}{l}0.57 \\
42\end{array}$ & $\begin{array}{l}0.64 \\
52\end{array}$ & $\begin{array}{l}1.03 \\
60\end{array}$ & $\begin{array}{l}1.00 \\
24\end{array}$ & $\begin{array}{l}0.62 \\
42\end{array}$ & $\begin{array}{l}3.254 \\
8\end{array}$ & $\begin{array}{l}1.04 \\
83\end{array}$ & $\begin{array}{l}0.65 \\
28\end{array}$ & $\begin{array}{l}1.177 \\
9\end{array}$ & $\begin{array}{l}1.06 \\
50\end{array}$ & $\begin{array}{l}0.66 \\
32\end{array}$ & 2.7898 & $\begin{array}{l}1 . \\
06 \\
59\end{array}$ & $\begin{array}{l}0.66 \\
38\end{array}$ & $\begin{array}{l}2.882 \\
8\end{array}$ & $\begin{array}{l}1.17 \\
64\end{array}$ & $\begin{array}{l}0.73 \\
26\end{array}$ & $\begin{array}{l}13.54 \\
61\end{array}$ \\
\hline $\begin{array}{l}54 . \\
5\end{array}$ & $\begin{array}{l}0.73 \\
35\end{array}$ & $\begin{array}{l}0.73 \\
25\end{array}$ & $\begin{array}{l}1.05 \\
50\end{array}$ & $\begin{array}{l}1.03 \\
02\end{array}$ & $\begin{array}{l}0.71 \\
52\end{array}$ & $\begin{array}{l}2.361 \\
7\end{array}$ & $\begin{array}{l}1.02 \\
92\end{array}$ & $\begin{array}{l}0.71 \\
45\end{array}$ & $\begin{array}{l}2.457 \\
3\end{array}$ & $\begin{array}{l}1.06 \\
35\end{array}$ & $\begin{array}{l}0.73 \\
83\end{array}$ & 0.7918 & $\begin{array}{l}0 . \\
98 \\
50\end{array}$ & $\begin{array}{l}0.68 \\
38\end{array}$ & $\begin{array}{l}6.648 \\
4\end{array}$ & $\begin{array}{l}1.15 \\
91\end{array}$ & $\begin{array}{l}0.80 \\
47\end{array}$ & $\begin{array}{l}9.856 \\
6\end{array}$ \\
\hline $\begin{array}{l}52 . \\
5\end{array}$ & $\begin{array}{l}0.81 \\
32\end{array}$ & $\begin{array}{l}0.78 \\
56\end{array}$ & $\begin{array}{l}1.09 \\
41\end{array}$ & $\begin{array}{l}1.11 \\
94\end{array}$ & $\begin{array}{l}0.80 \\
37\end{array}$ & $\begin{array}{l}2.303 \\
9\end{array}$ & $\begin{array}{l}1.06 \\
26\end{array}$ & $\begin{array}{l}0.76 \\
29\end{array}$ & $\begin{array}{l}2.889 \\
5\end{array}$ & $\begin{array}{l}1.10 \\
34\end{array}$ & $\begin{array}{l}0.79 \\
22\end{array}$ & 0.8401 & $\begin{array}{l}0 . \\
98 \\
39\end{array}$ & $\begin{array}{l}0.70 \\
64\end{array}$ & $\begin{array}{l}10.08 \\
14\end{array}$ & $\begin{array}{l}1.17 \\
50\end{array}$ & $\begin{array}{l}0.84 \\
36\end{array}$ & $\begin{array}{l}7.382 \\
8\end{array}$ \\
\hline $\begin{array}{l}51 . \\
5\end{array}$ & $\begin{array}{l}0.88 \\
45\end{array}$ & $\begin{array}{l}0.83 \\
54\end{array}$ & $\begin{array}{l}1.10 \\
79\end{array}$ & $\begin{array}{l}1.13 \\
91\end{array}$ & $\begin{array}{l}0.85 \\
88\end{array}$ & $\begin{array}{l}2.801 \\
0\end{array}$ & $\begin{array}{l}1.08 \\
14\end{array}$ & $\begin{array}{l}0.81 \\
53\end{array}$ & $\begin{array}{l}2.406 \\
0\end{array}$ & $\begin{array}{l}1.09 \\
76\end{array}$ & $\begin{array}{l}0.82 \\
75\end{array}$ & 0.9456 & $\begin{array}{l}0 . \\
98 \\
61\end{array}$ & $\begin{array}{l}0.74 \\
35\end{array}$ & $\begin{array}{l}11.00 \\
07\end{array}$ & $\begin{array}{l}1.16 \\
00\end{array}$ & $\begin{array}{l}0.87 \\
46\end{array}$ & $\begin{array}{l}4.692 \\
3\end{array}$ \\
\hline $\begin{array}{l}50 . \\
5\end{array}$ & $\begin{array}{l}0.94 \\
38\end{array}$ & $\begin{array}{l}0.89 \\
46\end{array}$ & $\begin{array}{l}1.15 \\
19\end{array}$ & $\begin{array}{l}1.17 \\
81\end{array}$ & $\begin{array}{l}0.91 \\
48\end{array}$ & $\begin{array}{l}2.257 \\
9\end{array}$ & $\begin{array}{l}1.12 \\
84\end{array}$ & $\begin{array}{l}0.87 \\
62\end{array}$ & $\begin{array}{l}2.056 \\
7\end{array}$ & $\begin{array}{l}1.14 \\
00\end{array}$ & $\begin{array}{l}0.88 \\
52\end{array}$ & 1.0507 & $\begin{array}{l}1 . \\
06 \\
33\end{array}$ & $\begin{array}{l}0.82 \\
57\end{array}$ & $\begin{array}{l}7.701 \\
7\end{array}$ & $\begin{array}{l}1.19 \\
27\end{array}$ & $\begin{array}{l}0.92 \\
62\end{array}$ & $\begin{array}{l}3.532 \\
3\end{array}$ \\
\hline $\begin{array}{l}50 . \\
5\end{array}$ & $\begin{array}{l}0.99 \\
78\end{array}$ & $\begin{array}{l}0.98 \\
37\end{array}$ & $\begin{array}{l}1.19 \\
81\end{array}$ & $\begin{array}{l}1.17 \\
99\end{array}$ & $\begin{array}{l}0.96 \\
87\end{array}$ & $\begin{array}{l}1.524 \\
8\end{array}$ & $\begin{array}{l}1.20 \\
41\end{array}$ & $\begin{array}{l}0.98 \\
85\end{array}$ & $\begin{array}{l}0.487 \\
9\end{array}$ & $\begin{array}{l}1.18 \\
82\end{array}$ & $\begin{array}{l}0.97 \\
55\end{array}$ & 0.8335 & $\begin{array}{l}1 . \\
14 \\
21\end{array}$ & $\begin{array}{l}0.93 \\
76\end{array}$ & $\begin{array}{l}4.686 \\
3\end{array}$ & $\begin{array}{l}1.19 \\
81\end{array}$ & $\begin{array}{l}0.98 \\
36\end{array}$ & $\begin{array}{l}0.010 \\
1\end{array}$ \\
\hline
\end{tabular}

Table 3: Experimental and Models Predicted VLE of Ethanol-water System at $101.325 \mathrm{kPa}$

\begin{tabular}{|c|c|c|c|c|c|c|c|c|c|c|c|c|c|c|c|c|c|c|}
\hline \multirow[b]{2}{*}{$\mathrm{T}$} & \multirow[b]{2}{*}{$X$} & \multirow[b]{2}{*}{$\mathrm{Y}$} & \multirow[b]{2}{*}{$\begin{array}{l}\gamma_{1} \\
\operatorname{Exp}\end{array}$} & \multicolumn{3}{|c|}{ NRTL } & \multicolumn{3}{|c|}{ UNIQUAC } & \multicolumn{3}{|c|}{ UNIFAC } & \multicolumn{3}{|l|}{ SRS } & \multicolumn{3}{|c|}{ TCRS } \\
\hline & & & & $\gamma_{1}$ & $\mathrm{y}_{1}$ & $\begin{array}{l}\mathrm{y}_{1} \\
\text { Error } \\
\%\end{array}$ & $\gamma_{1}$ & $\mathrm{y}_{1}$ & $\begin{array}{l}\mathrm{y}_{1} \\
\text { Error } \\
\%\end{array}$ & $\gamma_{1}$ & $\mathrm{y}_{1}$ & $\begin{array}{l}\mathrm{y}_{1} \\
\text { Error } \\
\%\end{array}$ & $\gamma_{1}$ & $\mathrm{y}_{1}$ & $\begin{array}{l}\mathrm{y}_{1} \\
\text { Error } \\
\%\end{array}$ & $\gamma_{1}$ & $\mathrm{y}_{1}$ & $\begin{array}{l}\mathrm{y}_{1} \\
\text { Error } \\
\%\end{array}$ \\
\hline $\begin{array}{l}92 . \\
5\end{array}$ & $\begin{array}{l}0.01 \\
70\end{array}$ & $\begin{array}{l}0.15 \\
89\end{array}$ & $\begin{array}{l}5.46 \\
44\end{array}$ & $\begin{array}{l}3.91 \\
41 \\
\end{array}$ & $\begin{array}{l}0.11 \\
38\end{array}$ & $\begin{array}{l}28.38 \\
26\end{array}$ & $\begin{array}{l}7.02 \\
09 \\
\end{array}$ & $\begin{array}{l}0.20 \\
41\end{array}$ & $\begin{array}{l}28.44 \\
55\end{array}$ & $\begin{array}{l}6.64 \\
02\end{array}$ & $\begin{array}{l}0.19 \\
3\end{array}$ & 21.46 & $\begin{array}{l}8.69 \\
51\end{array}$ & $\begin{array}{l}0.25 \\
28\end{array}$ & $\begin{array}{l}59.09 \\
37\end{array}$ & $\begin{array}{l}8.04 \\
01\end{array}$ & $\begin{array}{l}0.23 \\
37\end{array}$ & $\begin{array}{l}47.07 \\
36\end{array}$ \\
\hline $\begin{array}{l}90 . \\
5\end{array}$ & $\begin{array}{l}0.06 \\
21 \\
\end{array}$ & $\begin{array}{l}0.37 \\
89 \\
\end{array}$ & $\begin{array}{l}3.83 \\
60 \\
\end{array}$ & $\begin{array}{l}3.07 \\
97 \\
\end{array}$ & $\begin{array}{l}0.30 \\
42 \\
\end{array}$ & $\begin{array}{l}19.71 \\
70 \\
\end{array}$ & $\begin{array}{l}4.55 \\
59 \\
\end{array}$ & $\begin{array}{l}0.45 \\
00 \\
\end{array}$ & $\begin{array}{l}18.76 \\
17 \\
\end{array}$ & $\begin{array}{l}4.29 \\
68 \\
\end{array}$ & $\begin{array}{l}0.42 \\
44 \\
\end{array}$ & $\begin{array}{l}12.00 \\
54\end{array}$ & $\begin{array}{l}5.07 \\
71 \\
\end{array}$ & $\begin{array}{l}0.50 \\
15 \\
\end{array}$ & $\begin{array}{l}32.35 \\
33 \\
\end{array}$ & $\begin{array}{l}4.82 \\
21 \\
\end{array}$ & $\begin{array}{l}0.47 \\
63 \\
\end{array}$ & $\begin{array}{l}25.70 \\
26 \\
\end{array}$ \\
\hline $\begin{array}{l}88 . \\
5\end{array}$ & $\begin{array}{l}0.08 \\
56\end{array}$ & $\begin{array}{l}0.43 \\
75\end{array}$ & $\begin{array}{l}3.45 \\
86 \\
\end{array}$ & $\begin{array}{l}2.86 \\
59 \\
\end{array}$ & $\begin{array}{l}0.36 \\
25\end{array}$ & $\begin{array}{l}17.14 \\
28\end{array}$ & $\begin{array}{l}3.91 \\
73 \\
\end{array}$ & $\begin{array}{l}0.49 \\
55\end{array}$ & $\begin{array}{l}13.25 \\
71 \\
\end{array}$ & $\begin{array}{l}3.44 \\
76\end{array}$ & $\begin{array}{l}0.43 \\
61 \\
\end{array}$ & $\begin{array}{l}0.320 \\
0 \\
\end{array}$ & $\begin{array}{l}4.31 \\
89\end{array}$ & $\begin{array}{l}0.54 \\
63 \\
\end{array}$ & $\begin{array}{l}24.86 \\
85 \\
\end{array}$ & $\begin{array}{l}4.15 \\
62\end{array}$ & $\begin{array}{l}0.52 \\
57 \\
\end{array}$ & $\begin{array}{l}20.16 \\
00 \\
\end{array}$ \\
\hline $\begin{array}{l}86 . \\
0 \\
\end{array}$ & $\begin{array}{l}0.12 \\
38 \\
\end{array}$ & $\begin{array}{l}0.48 \\
05\end{array}$ & $\begin{array}{l}2.88 \\
34 \\
\end{array}$ & $\begin{array}{l}2.47 \\
78 \\
\end{array}$ & $\begin{array}{l}0.41 \\
29 \\
\end{array}$ & $\begin{array}{l}14.06 \\
86\end{array}$ & $\begin{array}{l}3.20 \\
57 \\
\end{array}$ & $\begin{array}{l}0.53 \\
42 \\
\end{array}$ & $\begin{array}{l}11.17 \\
58\end{array}$ & $\begin{array}{l}2.81 \\
35 \\
\end{array}$ & $\begin{array}{l}0.46 \\
88\end{array}$ & $\begin{array}{l}2.434 \\
9 \\
\end{array}$ & $\begin{array}{l}3.53 \\
82\end{array}$ & $\begin{array}{l}0.58 \\
96\end{array}$ & $\begin{array}{l}22.70 \\
55\end{array}$ & $\begin{array}{l}3.32 \\
34\end{array}$ & $\begin{array}{l}0.55 \\
38\end{array}$ & $\begin{array}{l}15.25 \\
49 \\
\end{array}$ \\
\hline $\begin{array}{l}84 . \\
5\end{array}$ & $\begin{array}{l}0.15 \\
61\end{array}$ & $\begin{array}{l}0.52 \\
19\end{array}$ & $\begin{array}{l}2.62 \\
87\end{array}$ & $\begin{array}{l}2.22 \\
94\end{array}$ & $\begin{array}{l}0.44 \\
26\end{array}$ & $\begin{array}{l}15.19 \\
44\end{array}$ & $\begin{array}{l}2.80 \\
06 \\
\end{array}$ & $\begin{array}{l}0.55 \\
60\end{array}$ & $\begin{array}{l}6.533 \\
8 \\
\end{array}$ & $\begin{array}{l}2.49 \\
03\end{array}$ & $\begin{array}{l}0.49 \\
44\end{array}$ & $\begin{array}{l}5.269 \\
2\end{array}$ & $\begin{array}{l}3.14 \\
22\end{array}$ & $\begin{array}{l}0.62 \\
38\end{array}$ & $\begin{array}{l}19.52 \\
48\end{array}$ & $\begin{array}{l}2.88 \\
99\end{array}$ & $\begin{array}{l}0.57 \\
37\end{array}$ & $\begin{array}{l}9.925 \\
2\end{array}$ \\
\hline $\begin{array}{l}84 . \\
5\end{array}$ & $\begin{array}{l}0.25 \\
08\end{array}$ & $\begin{array}{l}0.55 \\
8\end{array}$ & $\begin{array}{l}1.74 \\
93\end{array}$ & $\begin{array}{l}1.54 \\
31\end{array}$ & $\begin{array}{l}0.49 \\
22\end{array}$ & $\begin{array}{l}11.79 \\
21\end{array}$ & $\begin{array}{l}1.85 \\
38\end{array}$ & $\begin{array}{l}0.59 \\
13\end{array}$ & $\begin{array}{l}5.967 \\
7\end{array}$ & $\begin{array}{l}1.68 \\
73\end{array}$ & $\begin{array}{l}0.53 \\
82\end{array}$ & $\begin{array}{l}3.548 \\
8\end{array}$ & $\begin{array}{l}2.07 \\
29\end{array}$ & $\begin{array}{l}0.66 \\
12\end{array}$ & $\begin{array}{l}18.49 \\
46\end{array}$ & $\begin{array}{l}1.92 \\
06\end{array}$ & $\begin{array}{l}0.61 \\
26\end{array}$ & $\begin{array}{l}9.784 \\
9\end{array}$ \\
\hline $\begin{array}{l}82 . \\
5\end{array}$ & $\begin{array}{l}0.30 \\
75\end{array}$ & $\begin{array}{l}0.58 \\
26 \\
\end{array}$ & $\begin{array}{l}1.60 \\
81\end{array}$ & $\begin{array}{l}1.45 \\
61\end{array}$ & $\begin{array}{l}0.52 \\
75\end{array}$ & $\begin{array}{l}9.457 \\
6 \\
\end{array}$ & $\begin{array}{l}1.68 \\
13 \\
\end{array}$ & $\begin{array}{l}0.60 \\
91\end{array}$ & $\begin{array}{l}4.548 \\
5 \\
\end{array}$ & $\begin{array}{l}1.53 \\
86\end{array}$ & $\begin{array}{l}0.55 \\
74\end{array}$ & $\begin{array}{l}4.325 \\
4\end{array}$ & $\begin{array}{l}1.85 \\
74\end{array}$ & $\begin{array}{l}0.67 \\
29\end{array}$ & $\begin{array}{l}15.49 \\
94\end{array}$ & $\begin{array}{l}1.72 \\
99\end{array}$ & $\begin{array}{l}0.62 \\
67\end{array}$ & $\begin{array}{l}7.569 \\
5\end{array}$ \\
\hline $\begin{array}{l}80 . \\
5\end{array}$ & $\begin{array}{l}0.40 \\
65\end{array}$ & $\begin{array}{l}0.62 \\
23\end{array}$ & $\begin{array}{l}1.40 \\
40\end{array}$ & $\begin{array}{l}1.31 \\
36\end{array}$ & $\begin{array}{l}0.58 \\
22\end{array}$ & $\begin{array}{l}6.443 \\
8 \\
\end{array}$ & $\begin{array}{l}1.41 \\
79 \\
\end{array}$ & $\begin{array}{l}0.62 \\
84\end{array}$ & $\begin{array}{l}0.980 \\
2\end{array}$ & $\begin{array}{l}1.32 \\
72\end{array}$ & $\begin{array}{l}0.58 \\
82\end{array}$ & $\begin{array}{l}5.479 \\
6\end{array}$ & $\begin{array}{l}1.56 \\
27\end{array}$ & $\begin{array}{l}0.69 \\
26\end{array}$ & $\begin{array}{l}11.29 \\
68\end{array}$ & $\begin{array}{l}1.45 \\
19\end{array}$ & $\begin{array}{l}0.64 \\
35\end{array}$ & $\begin{array}{l}3.406 \\
7\end{array}$ \\
\hline $\begin{array}{l}79 . \\
5\end{array}$ & $\begin{array}{l}0.49 \\
79\end{array}$ & $\begin{array}{l}0.65 \\
64\end{array}$ & $\begin{array}{l}1.25 \\
73\end{array}$ & $\begin{array}{l}1.19 \\
42\end{array}$ & $\begin{array}{l}0.62 \\
34\end{array}$ & $\begin{array}{l}5.027 \\
4\end{array}$ & $\begin{array}{l}1.25 \\
09\end{array}$ & $\begin{array}{l}0.65 \\
11\end{array}$ & $\begin{array}{l}0.807 \\
4\end{array}$ & $\begin{array}{l}1.17 \\
27\end{array}$ & $\begin{array}{l}0.61 \\
22\end{array}$ & $\begin{array}{l}6.733 \\
6\end{array}$ & $\begin{array}{l}1.36 \\
85\end{array}$ & $\begin{array}{l}0.71 \\
44\end{array}$ & $\begin{array}{l}8.836 \\
0\end{array}$ & $\begin{array}{l}1.28 \\
44\end{array}$ & $\begin{array}{l}0.67 \\
05\end{array}$ & $\begin{array}{l}2.148 \\
0\end{array}$ \\
\hline $\begin{array}{l}79 . \\
0\end{array}$ & $\begin{array}{l}0.51 \\
98\end{array}$ & $\begin{array}{l}0.66 \\
29\end{array}$ & $\begin{array}{l}1.24 \\
04\end{array}$ & $\begin{array}{l}1.18 \\
42\end{array}$ & $\begin{array}{l}0.63 \\
28\end{array}$ & $\begin{array}{l}4.540 \\
6\end{array}$ & $\begin{array}{l}1.22 \\
55\end{array}$ & $\begin{array}{l}0.65 \\
49\end{array}$ & $\begin{array}{l}1.206 \\
8\end{array}$ & $\begin{array}{l}1.16 \\
57\end{array}$ & $\begin{array}{l}0.62 \\
29\end{array}$ & $\begin{array}{l}6.034 \\
0\end{array}$ & $\begin{array}{l}1.34 \\
77\end{array}$ & $\begin{array}{l}0.72 \\
02\end{array}$ & $\begin{array}{l}8.643 \\
8\end{array}$ & $\begin{array}{l}1.28 \\
09\end{array}$ & $\begin{array}{l}0.68 \\
45\end{array}$ & $\begin{array}{l}3.258 \\
4\end{array}$ \\
\hline $\begin{array}{l}78 . \\
0\end{array}$ & $\begin{array}{l}0.59 \\
23\end{array}$ & $\begin{array}{l}0.69 \\
21\end{array}$ & $\begin{array}{l}1.18 \\
23\end{array}$ & $\begin{array}{l}1.18 \\
33\end{array}$ & $\begin{array}{l}0.69 \\
26\end{array}$ & $\begin{array}{l}0.072 \\
2\end{array}$ & $\begin{array}{l}1.15 \\
82\end{array}$ & $\begin{array}{l}0.67 \\
79\end{array}$ & $\begin{array}{l}2.051 \\
7\end{array}$ & $\begin{array}{l}1.13 \\
28\end{array}$ & $\begin{array}{l}0.66 \\
3\end{array}$ & $\begin{array}{l}4.204 \\
5\end{array}$ & $\begin{array}{l}1.26 \\
49\end{array}$ & $\begin{array}{l}0.74 \\
04\end{array}$ & $\begin{array}{l}6.978 \\
7\end{array}$ & $\begin{array}{l}1.20 \\
19\end{array}$ & $\begin{array}{l}0.70 \\
35\end{array}$ & $\begin{array}{l}1.647 \\
1\end{array}$ \\
\hline $\begin{array}{l}77 . \\
0 \\
\end{array}$ & $\begin{array}{l}0.69 \\
63\end{array}$ & $\begin{array}{l}0.74 \\
95 \\
\end{array}$ & $\begin{array}{l}1.13 \\
33\end{array}$ & $\begin{array}{l}1.12 \\
33 \\
\end{array}$ & $\begin{array}{l}0.74 \\
28 \\
\end{array}$ & $\begin{array}{l}0.893 \\
9 \\
\end{array}$ & $\begin{array}{l}1.09 \\
77 \\
\end{array}$ & $\begin{array}{l}0.72 \\
59\end{array}$ & $\begin{array}{l}3.148 \\
7 \\
\end{array}$ & $\begin{array}{l}1.11 \\
2\end{array}$ & $\begin{array}{l}0.73 \\
53\end{array}$ & $\begin{array}{l}1.894 \\
5 \\
\end{array}$ & $\begin{array}{l}1.18 \\
35\end{array}$ & $\begin{array}{l}0.78 \\
26\end{array}$ & $\begin{array}{l}4.416 \\
2\end{array}$ & $\begin{array}{l}1.12 \\
71\end{array}$ & $\begin{array}{l}0.74 \\
53\end{array}$ & $\begin{array}{l}0.560 \\
3 \\
\end{array}$ \\
\hline $\begin{array}{l}75 . \\
5\end{array}$ & $\begin{array}{l}0.75 \\
72\end{array}$ & $\begin{array}{l}0.79 \\
65\end{array}$ & $\begin{array}{l}1.17 \\
62\end{array}$ & $\begin{array}{l}1.15 \\
58\end{array}$ & $\begin{array}{l}0.78 \\
26\end{array}$ & $\begin{array}{l}1.745 \\
1\end{array}$ & $\begin{array}{l}1.13 \\
67 \\
\end{array}$ & $\begin{array}{l}0.76 \\
97\end{array}$ & $\begin{array}{l}3.364 \\
7 \\
\end{array}$ & $\begin{array}{l}1.14 \\
16\end{array}$ & $\begin{array}{l}0.77 \\
3\end{array}$ & $\begin{array}{l}2.950 \\
4\end{array}$ & $\begin{array}{l}1.20 \\
03\end{array}$ & $\begin{array}{l}0.81 \\
27\end{array}$ & $\begin{array}{l}2.033 \\
8\end{array}$ & $\begin{array}{l}1.12 \\
90\end{array}$ & $\begin{array}{l}0.76 \\
45\end{array}$ & $\begin{array}{l}4.017 \\
5\end{array}$ \\
\hline $\begin{array}{l}74 . \\
5\end{array}$ & $\begin{array}{l}0.91 \\
43\end{array}$ & $\begin{array}{l}0.92 \\
43\end{array}$ & $\begin{array}{l}1.17 \\
70\end{array}$ & $\begin{array}{l}1.17 \\
59\end{array}$ & $\begin{array}{l}0.92 \\
33\end{array}$ & $\begin{array}{l}0.108 \\
1\end{array}$ & $\begin{array}{l}1.13 \\
19\end{array}$ & $\begin{array}{l}0.88 \\
88\end{array}$ & $\begin{array}{l}3.840 \\
7\end{array}$ & $\begin{array}{l}1.15 \\
55\end{array}$ & $\begin{array}{l}0.90 \\
73\end{array}$ & $\begin{array}{l}1.839 \\
2\end{array}$ & $\begin{array}{l}1.14 \\
34\end{array}$ & $\begin{array}{l}0.89 \\
78\end{array}$ & $\begin{array}{l}2.867 \\
0\end{array}$ & $\begin{array}{l}1.12 \\
92\end{array}$ & $\begin{array}{l}0.88 \\
67\end{array}$ & $\begin{array}{l}4.067 \\
9\end{array}$ \\
\hline
\end{tabular}

Table 4: Experimental and Models Predicted VLE of Ethanol-benzene System at $101.325 \mathrm{kPa}$

\begin{tabular}{|c|c|c|c|c|c|c|c|c|c|c|c|c|c|c|c|c|c|c|}
\hline \multirow[b]{2}{*}{$\mathrm{T}$} & \multirow[b]{2}{*}{$X$} & \multirow[b]{2}{*}{$\mathrm{Y}$} & \multirow[b]{2}{*}{$\begin{array}{l}\gamma_{1} \\
\operatorname{Exp}\end{array}$} & \multicolumn{3}{|c|}{ NRTL } & \multicolumn{3}{|c|}{ UNIQUAC } & \multicolumn{3}{|c|}{ UNIFAC } & \multicolumn{3}{|c|}{ SRS } & \multicolumn{3}{|c|}{ TCRS } \\
\hline & & & & $\gamma_{1}$ & $\mathrm{y}_{1}$ & $\begin{array}{l}\mathrm{y}_{1} \\
\text { Error } \\
\%\end{array}$ & $\gamma_{1}$ & $\mathrm{y}_{1}$ & $\begin{array}{l}\mathrm{y}_{1} \\
\text { Error } \\
\%\end{array}$ & $\gamma_{1}$ & $\mathrm{y}_{1}$ & $\begin{array}{ll}y_{1} & \text { Error } \\
\% & \end{array}$ & $\gamma_{1}$ & $\mathrm{y}_{1}$ & $\begin{array}{l}\mathrm{y}_{1} \\
\text { Erro } \\
\mathrm{r} \%\end{array}$ & $\gamma_{1}$ & $\mathrm{y}_{1}$ & $\begin{array}{l}\mathrm{y}_{1} \\
\text { Error } \\
\%\end{array}$ \\
\hline $\begin{array}{l}78 . \\
0\end{array}$ & $\begin{array}{l}0.01 \\
87\end{array}$ & $\begin{array}{l}0.12 \\
97\end{array}$ & $\begin{array}{l}7.09 \\
51\end{array}$ & $\begin{array}{l}8.03 \\
24\end{array}$ & $\begin{array}{l}0.14 \\
68\end{array}$ & $\begin{array}{l}13.18 \\
42\end{array}$ & $\begin{array}{l}11.15 \\
04\end{array}$ & $\begin{array}{l}0.20 \\
38\end{array}$ & $\begin{array}{l}57.13 \\
18\end{array}$ & $\begin{array}{l}2.95 \\
08\end{array}$ & $\begin{array}{l}0.05 \\
39\end{array}$ & 58.4117 & $\begin{array}{l}6 . \\
93 \\
91\end{array}$ & $\begin{array}{l}0.12 \\
68\end{array}$ & $\begin{array}{l}2.19 \\
52\end{array}$ & $\begin{array}{l}4.57 \\
36\end{array}$ & $\begin{array}{l}0.08 \\
36\end{array}$ & $\begin{array}{l}35.49 \\
73\end{array}$ \\
\hline $\begin{array}{l}74 . \\
0\end{array}$ & $\begin{array}{l}0.05 \\
23\end{array}$ & $\begin{array}{l}0.23 \\
94\end{array}$ & $\begin{array}{l}5.50 \\
72\end{array}$ & $\begin{array}{l}5.80 \\
86\end{array}$ & $\begin{array}{l}0.25 \\
25\end{array}$ & $\begin{array}{l}5.472 \\
0\end{array}$ & $\begin{array}{l}7.046 \\
5\end{array}$ & $\begin{array}{l}0.30 \\
63\end{array}$ & $\begin{array}{l}27.94 \\
48\end{array}$ & $\begin{array}{l}3.36 \\
35\end{array}$ & $\begin{array}{l}0.14 \\
62\end{array}$ & 38.9306 & $\begin{array}{l}6 . \\
04 \\
06\end{array}$ & $\begin{array}{l}0.26 \\
25\end{array}$ & $\begin{array}{l}9.68 \\
16\end{array}$ & $\begin{array}{l}4.50 \\
43\end{array}$ & $\begin{array}{l}0.19 \\
58\end{array}$ & $\begin{array}{l}18.21 \\
22\end{array}$ \\
\hline $\begin{array}{l}73 . \\
0\end{array}$ & $\begin{array}{l}0.09 \\
93\end{array}$ & $\begin{array}{l}0.30 \\
67\end{array}$ & $\begin{array}{l}3.87 \\
19\end{array}$ & $\begin{array}{l}4.20 \\
06\end{array}$ & $\begin{array}{l}0.33 \\
27\end{array}$ & $\begin{array}{l}8.477 \\
3\end{array}$ & $\begin{array}{l}4.870 \\
6\end{array}$ & $\begin{array}{l}0.38 \\
58\end{array}$ & $\begin{array}{l}25.79 \\
06\end{array}$ & $\begin{array}{l}2.88 \\
22\end{array}$ & $\begin{array}{l}0.22 \\
83\end{array}$ & 25.5624 & $\begin{array}{l}4 . \\
20 \\
06\end{array}$ & $\begin{array}{l}0.32 \\
98\end{array}$ & $\begin{array}{l}7.53 \\
80\end{array}$ & $\begin{array}{l}3.46 \\
69\end{array}$ & $\begin{array}{l}0.27 \\
46\end{array}$ & $\begin{array}{l}10.46 \\
62\end{array}$ \\
\hline $\begin{array}{l}68 . \\
5\end{array}$ & $\begin{array}{l}0.15 \\
67\end{array}$ & $\begin{array}{l}0.35 \\
96\end{array}$ & $\begin{array}{l}3.47 \\
88\end{array}$ & $\begin{array}{l}3.73 \\
35\end{array}$ & $\begin{array}{l}0.38 \\
58\end{array}$ & $\begin{array}{l}7.285 \\
8\end{array}$ & $\begin{array}{l}4.322 \\
5\end{array}$ & $\begin{array}{l}0.44 \\
68\end{array}$ & $\begin{array}{l}24.24 \\
91\end{array}$ & $\begin{array}{l}2.97 \\
39\end{array}$ & $\begin{array}{l}0.30 \\
74\end{array}$ & 14.5161 & $\begin{array}{l}3 . \\
44 \\
21\end{array}$ & $\begin{array}{l}0.35 \\
58\end{array}$ & $\begin{array}{l}1.05 \\
52\end{array}$ & $\begin{array}{l}3.43 \\
24\end{array}$ & $\begin{array}{l}0.35 \\
48\end{array}$ & $\begin{array}{l}1.334 \\
8\end{array}$ \\
\hline 66. & 0.23 & 0.39 & 2.81 & 2.96 & 0.42 & 5.314 & 3.424 & 0.48 & 21.80 & 2.63 & 0.37 & 6.29230 & 2. & 0.39 & 2.20 & 3.01 & 0.42 & 7.244 \\
\hline
\end{tabular}


Citation: Manojkumar Selvakumar and Sivaprakash Baskaran, 2018. Advers Thermodynamic Analysis of Azeotropic mixtures for Vapour liquid Equilibrium Determination-Experimental and Theoretical studies. Journal of Applied Sciences Research., 14(1): 12-24.

\begin{tabular}{|c|c|c|c|c|c|c|c|c|c|c|c|c|c|c|c|c|c|c|}
\hline 5 & 45 & 89 & 15 & 12 & 01 & 6 & 9 & 59 & 99 & 47 & 38 & & $\begin{array}{l}74 \\
97\end{array}$ & 01 & 46 & 54 & 78 & 9 \\
\hline $\begin{array}{l}66 . \\
0\end{array}$ & $\begin{array}{l}0.32 \\
41\end{array}$ & $\begin{array}{l}0.43 \\
22\end{array}$ & $\begin{array}{l}2.25 \\
27\end{array}$ & $\begin{array}{l}2.33 \\
51\end{array}$ & $\begin{array}{l}0.44 \\
79\end{array}$ & $\begin{array}{l}3.632 \\
5\end{array}$ & $\begin{array}{l}2.645 \\
3\end{array}$ & $\begin{array}{l}0.50 \\
75\end{array}$ & $\begin{array}{l}17.42 \\
24\end{array}$ & $\begin{array}{l}2.20 \\
07\end{array}$ & $\begin{array}{l}0.42 \\
22\end{array}$ & 2.31374 & $\begin{array}{l}2 . \\
17 \\
84\end{array}$ & $\begin{array}{l}0.41 \\
79\end{array}$ & $\begin{array}{l}3.30 \\
06\end{array}$ & $\begin{array}{l}2.47 \\
17\end{array}$ & $\begin{array}{l}0.47 \\
42\end{array}$ & $\begin{array}{l}9.717 \\
7\end{array}$ \\
\hline $\begin{array}{l}65 . \\
5\end{array}$ & $\begin{array}{l}0.45 \\
00\end{array}$ & $\begin{array}{l}0.45 \\
67\end{array}$ & $\begin{array}{l}1.75 \\
24\end{array}$ & $\begin{array}{l}1.76 \\
55\end{array}$ & $\begin{array}{l}0.46 \\
01\end{array}$ & $\begin{array}{l}0.744 \\
4\end{array}$ & $\begin{array}{l}2.002 \\
3\end{array}$ & $\begin{array}{l}0.52 \\
18\end{array}$ & $\begin{array}{l}14.25 \\
44\end{array}$ & $\begin{array}{l}1.84 \\
34\end{array}$ & $\begin{array}{l}0.48 \\
04\end{array}$ & 5.18940 & $\begin{array}{l}1 . \\
72 \\
74\end{array}$ & $\begin{array}{l}0.45 \\
01\end{array}$ & $\begin{array}{l}1.42 \\
84\end{array}$ & $\begin{array}{l}1.98 \\
54\end{array}$ & $\begin{array}{l}0.51 \\
74\end{array}$ & $\begin{array}{l}13.29 \\
10\end{array}$ \\
\hline $\begin{array}{l}64 . \\
5\end{array}$ & $\begin{array}{l}0.52 \\
28\end{array}$ & $\begin{array}{l}0.47 \\
80\end{array}$ & $\begin{array}{l}1.64 \\
98\end{array}$ & $\begin{array}{l}1.56 \\
81\end{array}$ & $\begin{array}{l}0.45 \\
43\end{array}$ & $\begin{array}{l}4.958 \\
1\end{array}$ & $\begin{array}{l}1.840 \\
5\end{array}$ & $\begin{array}{l}0.53 \\
32\end{array}$ & $\begin{array}{l}11.54 \\
81\end{array}$ & $\begin{array}{l}1.75 \\
14\end{array}$ & $\begin{array}{l}0.50 \\
74\end{array}$ & 6.15062 & $\begin{array}{l}1 . \\
63 \\
54\end{array}$ & $\begin{array}{l}0.47 \\
38\end{array}$ & $\begin{array}{l}0.86 \\
59\end{array}$ & $\begin{array}{l}1.87 \\
71\end{array}$ & $\begin{array}{l}0.54 \\
38\end{array}$ & $\begin{array}{l}13.76 \\
56\end{array}$ \\
\hline $\begin{array}{l}63 . \\
0\end{array}$ & $\begin{array}{l}0.71 \\
01\end{array}$ & $\begin{array}{l}0.52 \\
28\end{array}$ & $\begin{array}{l}1.42 \\
02\end{array}$ & $\begin{array}{l}1.33 \\
37\end{array}$ & $\begin{array}{l}0.49 \\
09\end{array}$ & $\begin{array}{l}6.101 \\
7\end{array}$ & $\begin{array}{l}1.537 \\
1\end{array}$ & $\begin{array}{l}0.56 \\
58\end{array}$ & $\begin{array}{l}8.224 \\
94\end{array}$ & $\begin{array}{l}1.57 \\
08\end{array}$ & $\begin{array}{l}0.57 \\
82\end{array}$ & 10.5967 & $\begin{array}{l}1 . \\
50 \\
56\end{array}$ & $\begin{array}{l}0.55 \\
42\end{array}$ & $\begin{array}{l}6.01 \\
60\end{array}$ & $\begin{array}{l}1.67 \\
01\end{array}$ & $\begin{array}{l}0.61 \\
47\end{array}$ & $\begin{array}{l}17.57 \\
84\end{array}$ \\
\hline $\begin{array}{l}62 . \\
0\end{array}$ & $\begin{array}{l}0.78 \\
66\end{array}$ & $\begin{array}{l}0.57 \\
60\end{array}$ & $\begin{array}{l}1.47 \\
75\end{array}$ & $\begin{array}{l}1.37 \\
04\end{array}$ & $\begin{array}{l}0.53 \\
42\end{array}$ & $\begin{array}{l}7.256 \\
9\end{array}$ & $\begin{array}{l}1.526 \\
6\end{array}$ & $\begin{array}{l}0.59 \\
51\end{array}$ & $\begin{array}{l}3.315 \\
97\end{array}$ & $\begin{array}{l}1.60 \\
43\end{array}$ & $\begin{array}{l}0.62 \\
54\end{array}$ & 8.57638 & $\begin{array}{l}1 . \\
54 \\
48\end{array}$ & $\begin{array}{l}0.60 \\
22\end{array}$ & $\begin{array}{l}4.56 \\
01\end{array}$ & $\begin{array}{l}1.67 \\
46\end{array}$ & $\begin{array}{l}0.65 \\
28\end{array}$ & $\begin{array}{l}13.33 \\
33\end{array}$ \\
\hline $\begin{array}{l}60 . \\
5\end{array}$ & $\begin{array}{l}0.87 \\
43\end{array}$ & $\begin{array}{l}0.68 \\
34\end{array}$ & $\begin{array}{l}1.68 \\
82\end{array}$ & $\begin{array}{l}1.60 \\
98\end{array}$ & $\begin{array}{l}0.65 \\
16\end{array}$ & $\begin{array}{l}4.653 \\
2\end{array}$ & $\begin{array}{l}1.616 \\
7\end{array}$ & $\begin{array}{l}0.65 \\
44\end{array}$ & $\begin{array}{l}4.243 \\
48\end{array}$ & $\begin{array}{l}1.79 \\
48\end{array}$ & $\begin{array}{l}0.72 \\
65\end{array}$ & 6.30670 & $\begin{array}{l}1 . \\
73 \\
33\end{array}$ & $\begin{array}{l}0.70 \\
16\end{array}$ & $\begin{array}{l}2.67 \\
31\end{array}$ & $\begin{array}{l}1.78 \\
96\end{array}$ & $\begin{array}{l}0.72 \\
44\end{array}$ & $\begin{array}{l}5.999 \\
4\end{array}$ \\
\hline $\begin{array}{l}60 . \\
0\end{array}$ & $\begin{array}{l}0.92 \\
30\end{array}$ & $\begin{array}{l}0.77 \\
34\end{array}$ & $\begin{array}{l}1.85 \\
16\end{array}$ & $\begin{array}{l}1.87 \\
16\end{array}$ & $\begin{array}{l}0.78 \\
17\end{array}$ & $\begin{array}{l}1.073 \\
1\end{array}$ & $\begin{array}{l}1.761 \\
0\end{array}$ & $\begin{array}{l}0.73 \\
55\end{array}$ & $\begin{array}{l}4.900 \\
44\end{array}$ & $\begin{array}{l}1.94 \\
79\end{array}$ & $\begin{array}{l}0.81 \\
36\end{array}$ & 5.19782 & $\begin{array}{l}1 . \\
87 \\
74\end{array}$ & $\begin{array}{l}0.78 \\
41\end{array}$ & $\begin{array}{l}1.39 \\
06\end{array}$ & $\begin{array}{l}1.95 \\
32\end{array}$ & $\begin{array}{l}0.81 \\
58\end{array}$ & $\begin{array}{l}5.482 \\
2\end{array}$ \\
\hline & & & Perce & $\mathrm{eE}$ & & $\begin{array}{l}5.697 \\
4\end{array}$ & & & $\begin{array}{l}18.40 \\
30\end{array}$ & & & $\begin{array}{l}15.67 \\
03\end{array}$ & & & $\begin{array}{l}3.57 \\
58\end{array}$ & & & $\begin{array}{l}12.66 \\
14\end{array}$ \\
\hline
\end{tabular}

Table 5: Experimental and Models Predicted VLE of Methanol-water System at $101.325 \mathrm{kPa}$

\begin{tabular}{|c|c|c|c|c|c|c|c|c|c|c|c|c|c|c|c|c|c|c|}
\hline \multirow[b]{2}{*}{$\mathrm{T}$} & \multirow[b]{2}{*}{$\mathrm{X}$} & \multirow[b]{2}{*}{$\mathrm{Y}$} & \multirow[b]{2}{*}{$\begin{array}{l}\gamma_{1} \\
\operatorname{Exp}\end{array}$} & \multicolumn{3}{|c|}{ NRTL } & \multicolumn{3}{|c|}{ UNIQUAC } & \multicolumn{3}{|c|}{ UNIFAC } & \multicolumn{3}{|c|}{ SRS } & \multicolumn{3}{|c|}{ TCRS } \\
\hline & & & & $\gamma_{1}$ & $\mathrm{y}_{1}$ & $\begin{array}{l}\mathrm{y}_{1} \\
\text { Error } \\
\%\end{array}$ & $\gamma_{1}$ & $\mathrm{y}_{1}$ & $\begin{array}{l}\mathrm{y}_{1} \\
\text { Error } \\
\%\end{array}$ & $\gamma_{1}$ & $\mathrm{y}_{1}$ & $\begin{array}{l}\mathrm{y}_{1} \text { Error } \\
\%\end{array}$ & $\gamma_{1}$ & $\mathrm{y}_{1}$ & $\begin{array}{l}\mathrm{y}_{1} \\
\text { Error } \\
\%\end{array}$ & $\gamma_{1}$ & $\mathrm{y}_{1}$ & $\begin{array}{l}\mathrm{y}_{1} \\
\text { Error } \\
\%\end{array}$ \\
\hline $\begin{array}{l}96 . \\
4\end{array}$ & $\begin{array}{l}0.01 \\
89\end{array}$ & $\begin{array}{l}0.12 \\
34\end{array}$ & $\begin{array}{l}1.86 \\
95\end{array}$ & $\begin{array}{l}2.66 \\
41\end{array}$ & $\begin{array}{l}0.17 \\
58\end{array}$ & $\begin{array}{l}42.46 \\
35\end{array}$ & $\begin{array}{l}2.04 \\
71\end{array}$ & $\begin{array}{l}0.13 \\
51\end{array}$ & $\begin{array}{l}9.481 \\
3\end{array}$ & $\begin{array}{l}2.01 \\
32\end{array}$ & $\begin{array}{l}0.13 \\
28\end{array}$ & 7.6175 & $\begin{array}{l}1 . \\
14 \\
09\end{array}$ & $\begin{array}{l}0.07 \\
53\end{array}$ & $\begin{array}{l}38.97 \\
89\end{array}$ & $\begin{array}{l}0.72 \\
42\end{array}$ & $\begin{array}{l}0.04 \\
78\end{array}$ & $\begin{array}{l}61.26 \\
41\end{array}$ \\
\hline $\begin{array}{l}93 . \\
5\end{array}$ & $\begin{array}{l}0.03 \\
89\end{array}$ & $\begin{array}{l}0.22 \\
30\end{array}$ & $\begin{array}{l}1.84 \\
32\end{array}$ & $\begin{array}{l}2.30 \\
48\end{array}$ & $\begin{array}{l}0.27 \\
88\end{array}$ & $\begin{array}{l}25.02 \\
24\end{array}$ & $\begin{array}{l}1.82 \\
34\end{array}$ & $\begin{array}{l}0.22 \\
06\end{array}$ & $\begin{array}{l}1.076 \\
2\end{array}$ & $\begin{array}{l}1.66 \\
67\end{array}$ & $\begin{array}{l}0.20 \\
16\end{array}$ & 9.5964 & $\begin{array}{l}1 . \\
50 \\
97\end{array}$ & $\begin{array}{l}0.18 \\
26\end{array}$ & $\begin{array}{l}18.11 \\
65\end{array}$ & $\begin{array}{l}1.18 \\
71\end{array}$ & $\begin{array}{l}0.14 \\
36\end{array}$ & $\begin{array}{l}35.60 \\
53\end{array}$ \\
\hline $\begin{array}{l}91 . \\
2\end{array}$ & $\begin{array}{l}0.05 \\
88\end{array}$ & $\begin{array}{l}0.31 \\
04\end{array}$ & $\begin{array}{l}1.86 \\
52\end{array}$ & $\begin{array}{l}2.14 \\
06\end{array}$ & $\begin{array}{l}0.35 \\
62\end{array}$ & $\begin{array}{l}14.75 \\
51\end{array}$ & $\begin{array}{l}1.91 \\
51\end{array}$ & $\begin{array}{l}0.31 \\
87\end{array}$ & $\begin{array}{l}2.673 \\
9\end{array}$ & $\begin{array}{l}1.85 \\
75\end{array}$ & $\begin{array}{l}0.30 \\
91\end{array}$ & 0.4188 & $\begin{array}{l}1 . \\
70 \\
49\end{array}$ & $\begin{array}{l}0.28 \\
37\end{array}$ & $\begin{array}{l}8.601 \\
8\end{array}$ & $\begin{array}{l}1.47 \\
11\end{array}$ & $\begin{array}{l}0.24 \\
48\end{array}$ & $\begin{array}{l}21.13 \\
40\end{array}$ \\
\hline $\begin{array}{l}89 . \\
3\end{array}$ & $\begin{array}{l}0.08 \\
27\end{array}$ & $\begin{array}{l}0.36 \\
65\end{array}$ & $\begin{array}{l}1.68 \\
88\end{array}$ & $\begin{array}{l}1.86 \\
55\end{array}$ & $\begin{array}{l}0.40 \\
48\end{array}$ & $\begin{array}{l}10.45 \\
02\end{array}$ & $\begin{array}{l}1.80 \\
75\end{array}$ & $\begin{array}{l}0.39 \\
22\end{array}$ & $\begin{array}{l}7.012 \\
2\end{array}$ & $\begin{array}{l}1.71 \\
38\end{array}$ & $\begin{array}{l}0.37 \\
19\end{array}$ & 1.4733 & $\begin{array}{l}1 . \\
71 \\
15\end{array}$ & $\begin{array}{l}0.31 \\
74\end{array}$ & $\begin{array}{l}13.39 \\
70\end{array}$ & $\begin{array}{l}1.30 \\
69\end{array}$ & $\begin{array}{l}0.28 \\
36\end{array}$ & $\begin{array}{l}22.61 \\
93\end{array}$ \\
\hline $\begin{array}{l}87 . \\
7\end{array}$ & $\begin{array}{l}0.10 \\
32\end{array}$ & $\begin{array}{l}0.42 \\
28\end{array}$ & $\begin{array}{l}1.66 \\
43\end{array}$ & $\begin{array}{l}1.80 \\
61\end{array}$ & $\begin{array}{l}0.45 \\
88\end{array}$ & $\begin{array}{l}8.514 \\
6\end{array}$ & $\begin{array}{l}1.83 \\
26\end{array}$ & $\begin{array}{l}0.46 \\
55\end{array}$ & $\begin{array}{l}10.09 \\
93\end{array}$ & $\begin{array}{l}1.67 \\
04\end{array}$ & $\begin{array}{l}0.42 \\
43\end{array}$ & 0.3548 & $\begin{array}{l}1 . \\
54 \\
63 \\
\end{array}$ & $\begin{array}{l}0.39 \\
28\end{array}$ & $\begin{array}{l}7.095 \\
5\end{array}$ & $\begin{array}{l}1.42 \\
55\end{array}$ & $\begin{array}{l}0.36 \\
21\end{array}$ & $\begin{array}{l}14.35 \\
66\end{array}$ \\
\hline $\begin{array}{l}84 . \\
4\end{array}$ & $\begin{array}{l}0.14 \\
85\end{array}$ & $\begin{array}{l}0.51 \\
17\end{array}$ & $\begin{array}{l}1.47 \\
77\end{array}$ & $\begin{array}{l}1.66 \\
43\end{array}$ & $\begin{array}{l}0.57 \\
63\end{array}$ & $\begin{array}{l}12.62 \\
45\end{array}$ & $\begin{array}{l}1.63 \\
23\end{array}$ & $\begin{array}{l}0.56 \\
52\end{array}$ & $\begin{array}{l}10.45 \\
53\end{array}$ & $\begin{array}{l}1.50 \\
58\end{array}$ & $\begin{array}{l}0.52 \\
14\end{array}$ & 1.8956 & $\begin{array}{l}1 . \\
39 \\
24 \\
\end{array}$ & $\begin{array}{l}0.48 \\
21\end{array}$ & $\begin{array}{l}5.784 \\
6\end{array}$ & $\begin{array}{l}1.33 \\
66\end{array}$ & $\begin{array}{l}0.46 \\
28\end{array}$ & $\begin{array}{l}9.556 \\
3\end{array}$ \\
\hline $\begin{array}{l}81 . \\
7\end{array}$ & $\begin{array}{l}0.18 \\
97\end{array}$ & $\begin{array}{l}0.57 \\
29\end{array}$ & $\begin{array}{l}1.45 \\
04\end{array}$ & $\begin{array}{l}1.60 \\
73\end{array}$ & $\begin{array}{l}0.63 \\
48\end{array}$ & $\begin{array}{l}10.80 \\
46\end{array}$ & $\begin{array}{l}1.56 \\
52\end{array}$ & $\begin{array}{l}0.61 \\
82\end{array}$ & $\begin{array}{l}7.907 \\
1\end{array}$ & $\begin{array}{l}1.48 \\
37\end{array}$ & $\begin{array}{l}0.58 \\
60\end{array}$ & 2.2866 & $\begin{array}{l}1 . \\
36 \\
27\end{array}$ & $\begin{array}{l}0.53 \\
82\end{array}$ & $\begin{array}{l}6.056 \\
9\end{array}$ & $\begin{array}{l}1.28 \\
65\end{array}$ & $\begin{array}{l}0.50 \\
81\end{array}$ & $\begin{array}{l}11.31 \\
08\end{array}$ \\
\hline $\begin{array}{l}78 . \\
0\end{array}$ & $\begin{array}{l}0.30 \\
16\end{array}$ & $\begin{array}{l}0.67 \\
65\end{array}$ & $\begin{array}{l}1.18 \\
39\end{array}$ & $\begin{array}{l}1.27 \\
10\end{array}$ & $\begin{array}{l}0.72 \\
62\end{array}$ & $\begin{array}{l}7.346 \\
6\end{array}$ & $\begin{array}{l}1.22 \\
95\end{array}$ & $\begin{array}{l}0.70 \\
25\end{array}$ & $\begin{array}{l}3.843 \\
3\end{array}$ & $\begin{array}{l}1.18 \\
72\end{array}$ & $\begin{array}{l}0.67 \\
83\end{array}$ & 0.2660 & $\begin{array}{l}1 . \\
11 \\
59\end{array}$ & $\begin{array}{l}0.63 \\
76\end{array}$ & $\begin{array}{l}5.750 \\
1\end{array}$ & $\begin{array}{l}1.09 \\
2\end{array}$ & $\begin{array}{l}0.62 \\
39\end{array}$ & $\begin{array}{l}7.775 \\
3\end{array}$ \\
\hline $\begin{array}{l}75 . \\
3\end{array}$ & $\begin{array}{l}0.39 \\
82\end{array}$ & $\begin{array}{l}0.73 \\
11\end{array}$ & $\begin{array}{l}1.10 \\
58\end{array}$ & $\begin{array}{l}1.16 \\
19\end{array}$ & $\begin{array}{l}0.76 \\
81\end{array}$ & $\begin{array}{l}5.060 \\
8\end{array}$ & $\begin{array}{l}1.12 \\
72\end{array}$ & $\begin{array}{l}0.74 \\
52\end{array}$ & $\begin{array}{l}1.928 \\
6\end{array}$ & $\begin{array}{l}1.12 \\
39\end{array}$ & $\begin{array}{l}0.74 \\
30\end{array}$ & 1.6276 & $\begin{array}{l}1 . \\
06 \\
28 \\
\end{array}$ & $\begin{array}{l}0.70 \\
26\end{array}$ & $\begin{array}{l}3.898 \\
2\end{array}$ & $\begin{array}{l}1.05 \\
01\end{array}$ & $\begin{array}{l}0.69 \\
42\end{array}$ & $\begin{array}{l}5.047 \\
1\end{array}$ \\
\hline $\begin{array}{l}72 . \\
1\end{array}$ & $\begin{array}{l}0.51 \\
20\end{array}$ & $\begin{array}{l}0.78 \\
19\end{array}$ & $\begin{array}{l}1.01 \\
48\end{array}$ & $\begin{array}{l}1.05 \\
73\end{array}$ & $\begin{array}{l}0.81 \\
46\end{array}$ & $\begin{array}{l}4.182 \\
1\end{array}$ & $\begin{array}{l}1.03 \\
28\end{array}$ & $\begin{array}{l}0.79 \\
45\end{array}$ & $\begin{array}{l}1.611 \\
4\end{array}$ & $\begin{array}{l}1.03 \\
15\end{array}$ & $\begin{array}{l}0.79 \\
47\end{array}$ & 1.6370 & $\begin{array}{l}0 . \\
99 \\
14 \\
\end{array}$ & $\begin{array}{l}0.76 \\
38\end{array}$ & $\begin{array}{l}2.314 \\
8\end{array}$ & $\begin{array}{l}1.00 \\
31\end{array}$ & $\begin{array}{l}0.77 \\
28\end{array}$ & $\begin{array}{l}1.163 \\
8\end{array}$ \\
\hline $\begin{array}{l}67 . \\
2\end{array}$ & $\begin{array}{l}0.60 \\
15\end{array}$ & $\begin{array}{l}0.83 \\
05\end{array}$ & $\begin{array}{l}1.03 \\
31\end{array}$ & $\begin{array}{l}1.05 \\
91\end{array}$ & $\begin{array}{l}0.85 \\
13\end{array}$ & $\begin{array}{l}2.504 \\
5\end{array}$ & $\begin{array}{l}1.04 \\
35\end{array}$ & $\begin{array}{l}0.83 \\
88\end{array}$ & $\begin{array}{l}0.999 \\
3\end{array}$ & $\begin{array}{l}1.04 \\
59\end{array}$ & $\begin{array}{l}0.84 \\
07\end{array}$ & 1.2281 & $\begin{array}{l}1 . \\
01 \\
11 \\
\end{array}$ & $\begin{array}{l}0.81 \\
27\end{array}$ & $\begin{array}{l}2.143 \\
2\end{array}$ & $\begin{array}{l}1.02 \\
22\end{array}$ & $\begin{array}{l}0.82 \\
17\end{array}$ & $\begin{array}{l}1.059 \\
6\end{array}$ \\
\hline $\begin{array}{l}66 . \\
3\end{array}$ & $\begin{array}{l}0.71 \\
27\end{array}$ & $\begin{array}{l}0.86 \\
89\end{array}$ & $\begin{array}{l}1.09 \\
93\end{array}$ & $\begin{array}{l}1.11 \\
16\end{array}$ & $\begin{array}{l}0.87 \\
86\end{array}$ & $\begin{array}{l}1.116 \\
3\end{array}$ & $\begin{array}{l}1.10 \\
81\end{array}$ & $\begin{array}{l}0.87 \\
58\end{array}$ & $\begin{array}{l}0.794 \\
1\end{array}$ & $\begin{array}{l}1.11 \\
12\end{array}$ & $\begin{array}{l}0.87 \\
82\end{array}$ & 1.0703 & $\begin{array}{l}1 . \\
07 \\
90\end{array}$ & $\begin{array}{l}0.85 \\
28\end{array}$ & $\begin{array}{l}1.852 \\
9\end{array}$ & $\begin{array}{l}1.08 \\
96\end{array}$ & $\begin{array}{l}0.86 \\
12\end{array}$ & $\begin{array}{l}0.886 \\
1\end{array}$ \\
\hline $\begin{array}{l}64 . \\
5\end{array}$ & $\begin{array}{l}0.82 \\
57\end{array}$ & $\begin{array}{l}0.92 \\
11\end{array}$ & $\begin{array}{l}1.04 \\
16\end{array}$ & $\begin{array}{l}1.05 \\
22\end{array}$ & $\begin{array}{l}0.93 \\
04\end{array}$ & $\begin{array}{l}1.004 \\
1\end{array}$ & $\begin{array}{l}1.03 \\
12\end{array}$ & $\begin{array}{l}0.91 \\
18\end{array}$ & $\begin{array}{l}1.015 \\
0\end{array}$ & $\begin{array}{l}1.03 \\
32\end{array}$ & $\begin{array}{l}0.91 \\
36\end{array}$ & 0.8196 & $\begin{array}{l}1 . \\
02 \\
95\end{array}$ & $\begin{array}{l}0.91 \\
03\end{array}$ & $\begin{array}{l}1.177 \\
8\end{array}$ & $\begin{array}{l}.03 \\
06\end{array}$ & $\begin{array}{l}0.91 \\
13\end{array}$ & $\begin{array}{l}1.069 \\
3\end{array}$ \\
\hline & & & Error & centa & & $\begin{array}{l}11.21 \\
91\end{array}$ & & & $\begin{array}{l}4.530 \\
5\end{array}$ & & & $\begin{array}{l}2.33 \\
01\end{array}$ & & & $\begin{array}{l}8.859 \\
1\end{array}$ & & & $\begin{array}{l}14.83 \\
44\end{array}$ \\
\hline
\end{tabular}

Modelling:

Generally distillation columns are operated under varying conditions of temperature and pressure depending on the feed conditions and the desired quality of products. Obtaining VLE for such varying conditions experimentally is quite complex and expensive too. Hence theoretical estimation of VLE using activity coefficient models becomes inevitable. In this context five models namely NRTL, UNIQUAC, UNIFAC (Rao, Y.V.C., 1997) and two modified forms of Florry Huggins' model namely SRS and TCRS (Sabarathinam, P.L., B. Sivaprakash, 2002) were employed in the present study. Newton Raphson technique was used to 

Determination-Experimental and Theoretical studies. Journal of Applied Sciences Research., 14(1): 12-24.

make the computations with computer programming in Java software of 1.6 version. The parameters estimated are presented in table 6 . Binary interaction parameters for UNIFAC method have been taken from the literature (Rao, Y.V.C., 1997).

Table 6: Estimated NRTL, UNIQUAC, SRC and TCRS parameters of five azeotropic systems

\begin{tabular}{lllllllll}
\hline \multirow{2}{*}{ System } & $\mathrm{NRTL} \mathrm{J} / \mathrm{mol} \mathrm{K}$ & \multicolumn{3}{c}{ UNIQUAC } & $\mathrm{J} / \mathrm{mol} \mathrm{K}$ & \multicolumn{2}{l}{$\mathrm{SRS} \mathrm{J} / \mathrm{mol} \mathrm{K}$} & \multicolumn{2}{c}{ TCRS J/mol K } \\
\cline { 2 - 9 } & $\lambda_{12}-\lambda_{11}$ & $\lambda_{21}-\lambda_{22}$ & $\mathrm{u}_{12}-\mathrm{u}_{22}$ & $\mathrm{u}_{21}-\mathrm{u}_{11}$ & $\lambda_{12}-\lambda_{11}$ & $\lambda_{21}-\lambda_{22}$ & $\lambda_{12}-\lambda_{11}$ & $\lambda_{21}-\lambda_{22}$ \\
\hline Acetone-water & 2160.1319 & 18602.345 & -580.1319 & 1112.3450 & 3066.913 & 29.5959 & 5326.9130 & -2230.4040 \\
Acetone- & -17460.218 & -51385.655 & 4769.778 & 19434.015 & 1646.530 & -1633.5299 & 8046.530 & -8033.5299 \\
methanol & -1835.131 & 12920.345 & -860.1319 & 1392.345 & -1232.8499 & 3400.00 & -1852.849 & 2780.00 \\
Ethanol-water & -1920.1319 & 9922.3450 & 320.1319 & 852.3450 & 167.1500 & 4800.00 & 132.8499 & 4500.00 \\
Ethanol-benzene & \multirow{2}{*}{1542.3499} & 12399.868 & -11867.654 & 21832.32 & -16828.438 & 12980.279 & -16430.404 \\
Methanol -water & 699.8679 & & & & & & &
\end{tabular}

The complete comparison of VLE predicted from the five models with the experimental data is presented in tables 1-5. The experimental and correlated $\mathrm{x}-\mathrm{y}$ diagrams of five azeotropic systems using the five models are given in figures 2-6.

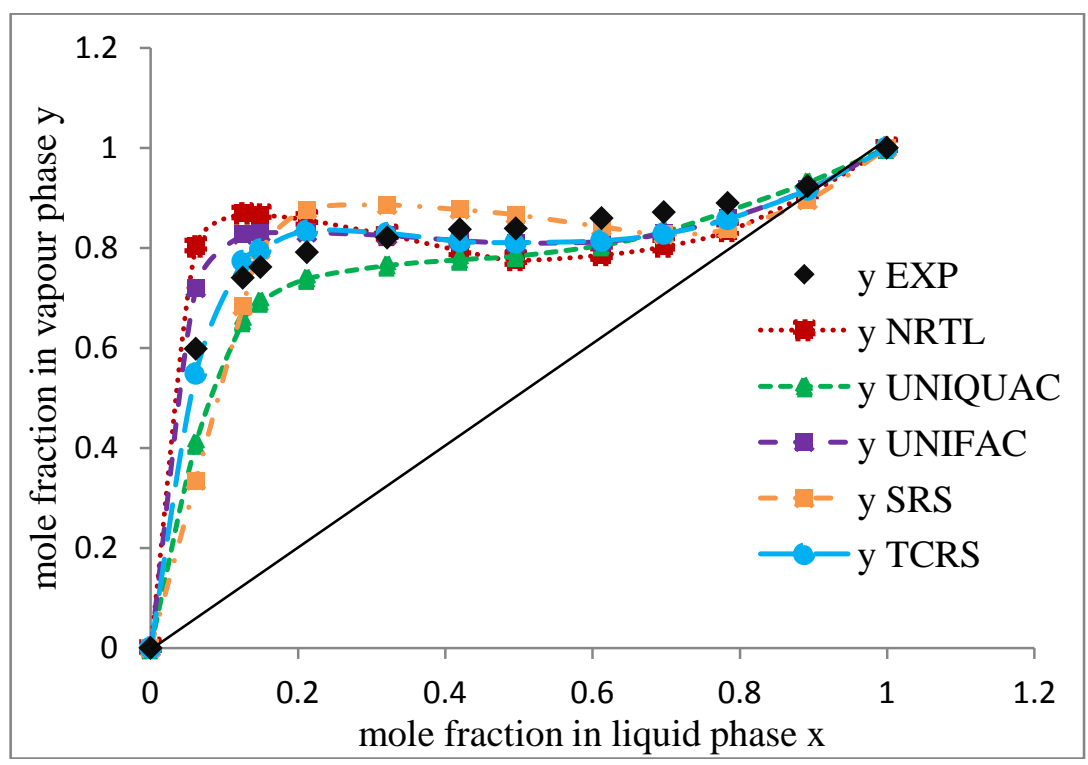

Fig. 2: Experimental and Correlated xy Diagram of Acetone-water System at $101.325 \mathrm{kPa}$.

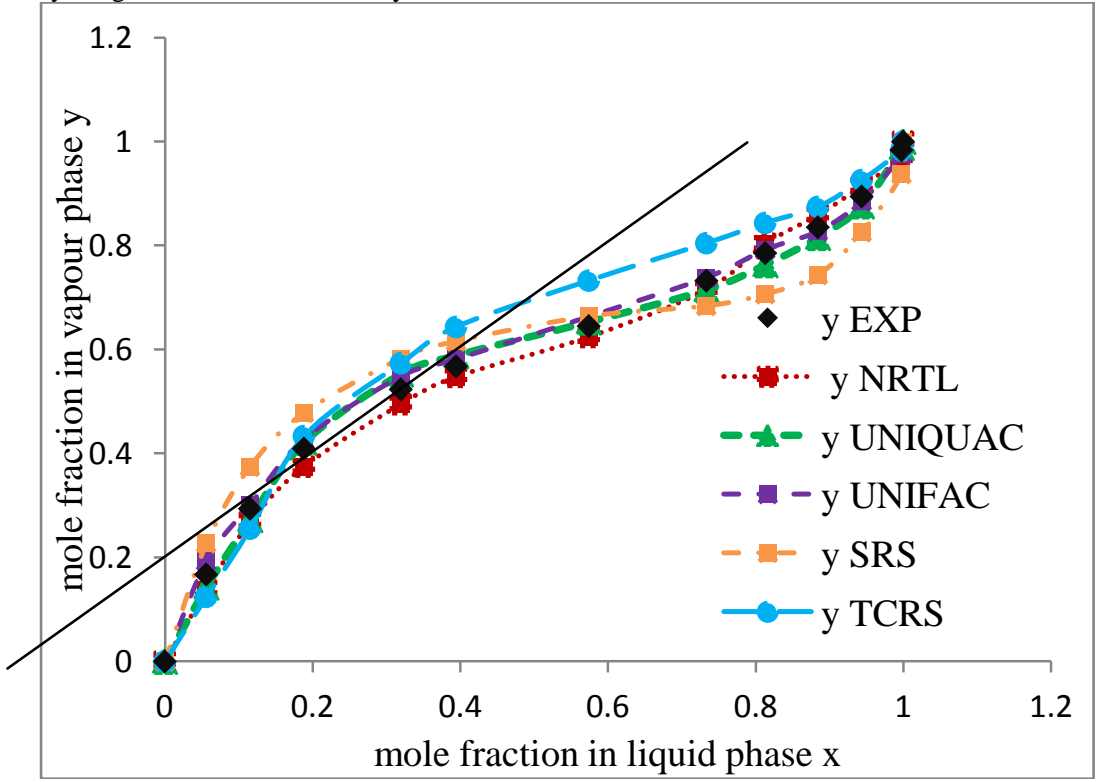

Fig. 3: Experimental and Correlated xy Diagram of Acetone-methanol System at $101.325 \mathrm{kPa}$. 


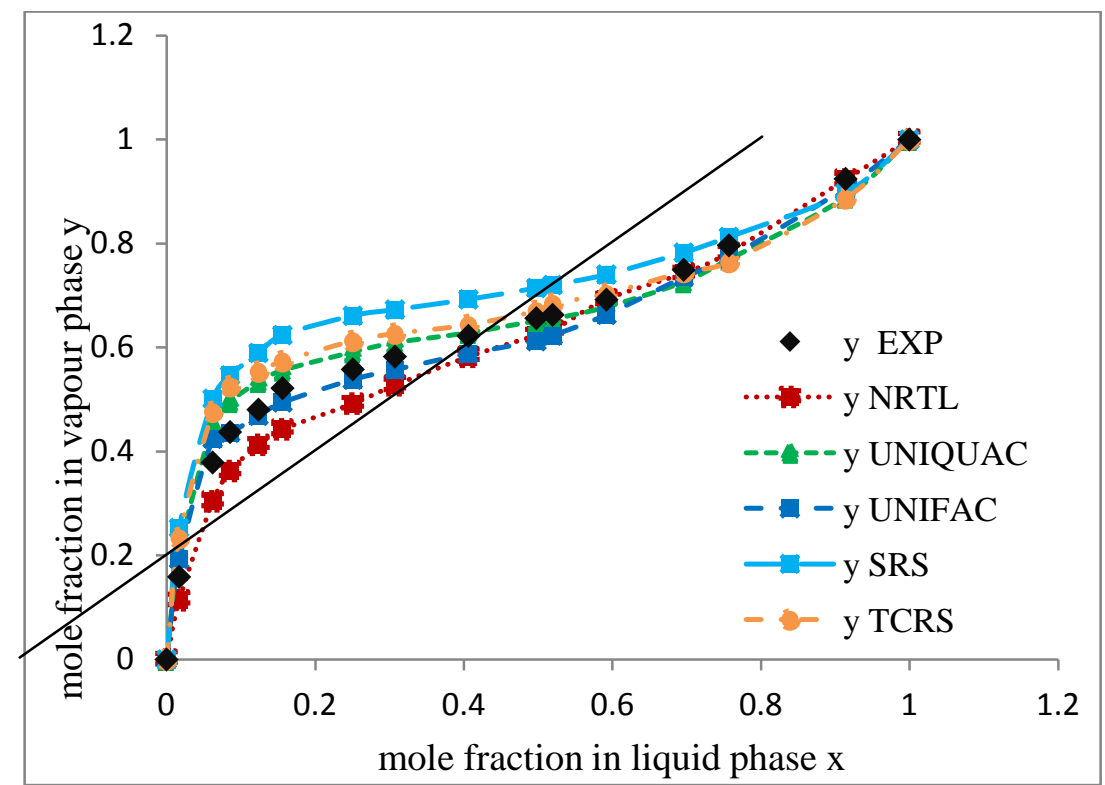

Fig. 4: Experimental and Correlated xy Diagram of Ethanol-water System at $101.325 \mathrm{kPa}$.

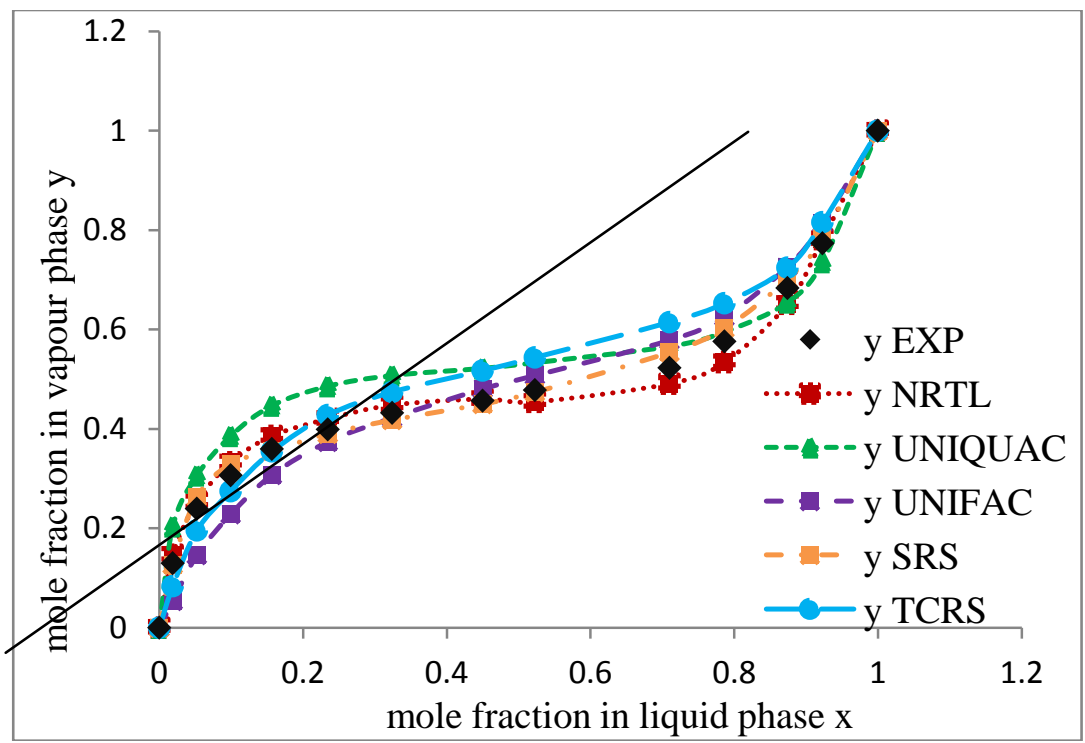

Fig. 5: Experimental and Correlated xy Diagram of Ethanol-benzene System at $101.325 \mathrm{kPa}$.

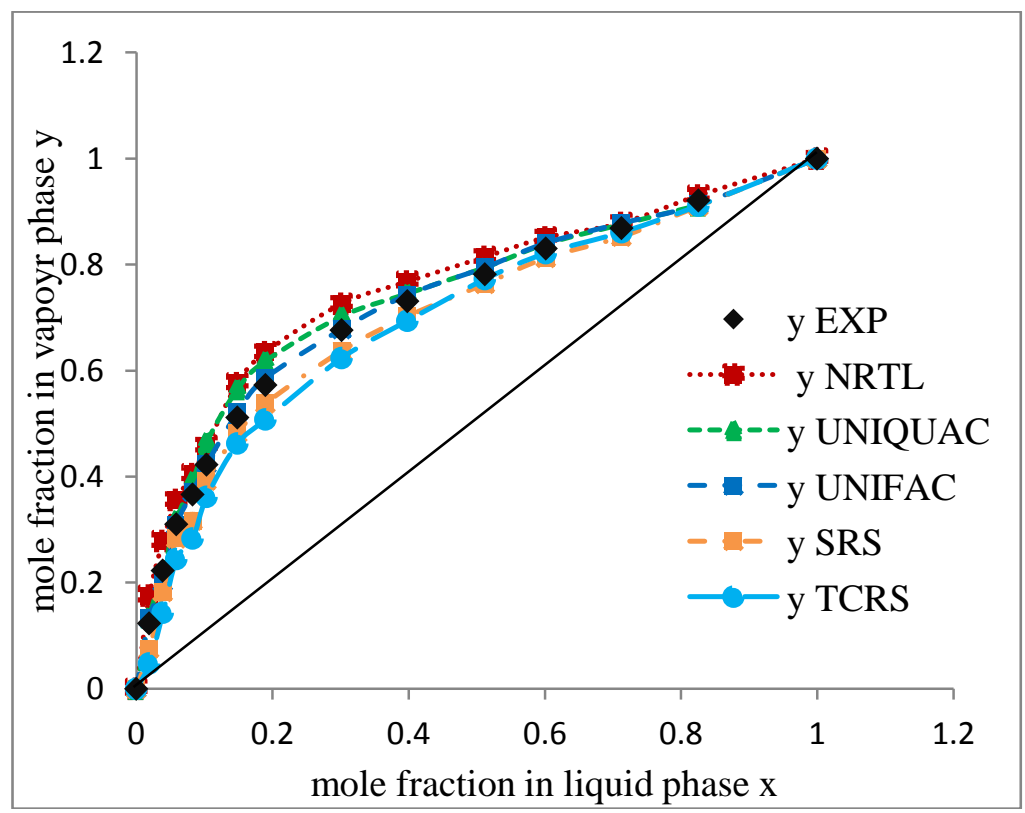

Fig. 6: Experimental and Correlated xy Diagram of Methanol-water System at $101.325 \mathrm{kPa}$. 
The overall error percentages of the VLE for acetone-water system using five activity coefficient models (NRTL, UNIQUAC, UNIFAC, SRS and TCRS) are $7.9438,8.4590,6.0952,8.9785$, and 4.0380 respectively as indicated in table 1. It is observed that TCRS model have lesser error percentages than the other four models. Acetone-methanol system shows significant validity for the UNIFAC and UNIQUAC models with the least error percentage of 3.3167 and 4.1357 whereas the other models have more than $5 \%$ (table 2.) Error occurred in UNIFAC model (5.6071\%) provide good representation of VLE for ethanol-water system when compared to other models (table 3). The SRS and NRTL model gave better results for the ethanol-benzene system yielding an error percentage of 3.5758 and 5.6974 (table 4). Of all the five models chosen, the UNIFAC and UNIQUAC models gives better result for methanol-water system with least error percentage of 2.3301 and 4.5305 (table 5).

Related outcome were observed with the Redlich-Kister method of thermodynamics consistency test (Eq. 32). This can be seen from the figures 7-11.

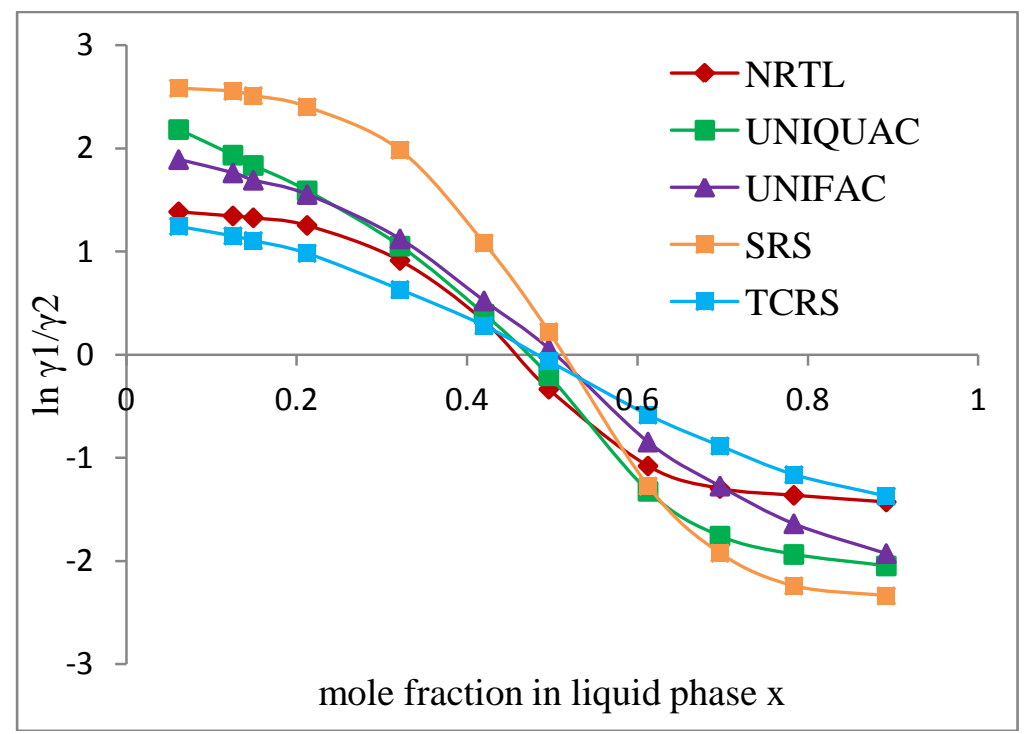

Fig. 7: Thermodynamic Consistency test of Acetone-water System Using Redlich Kiester method for NRTL, UNIQUAC, UNIFAC, SRS and TCRS models.

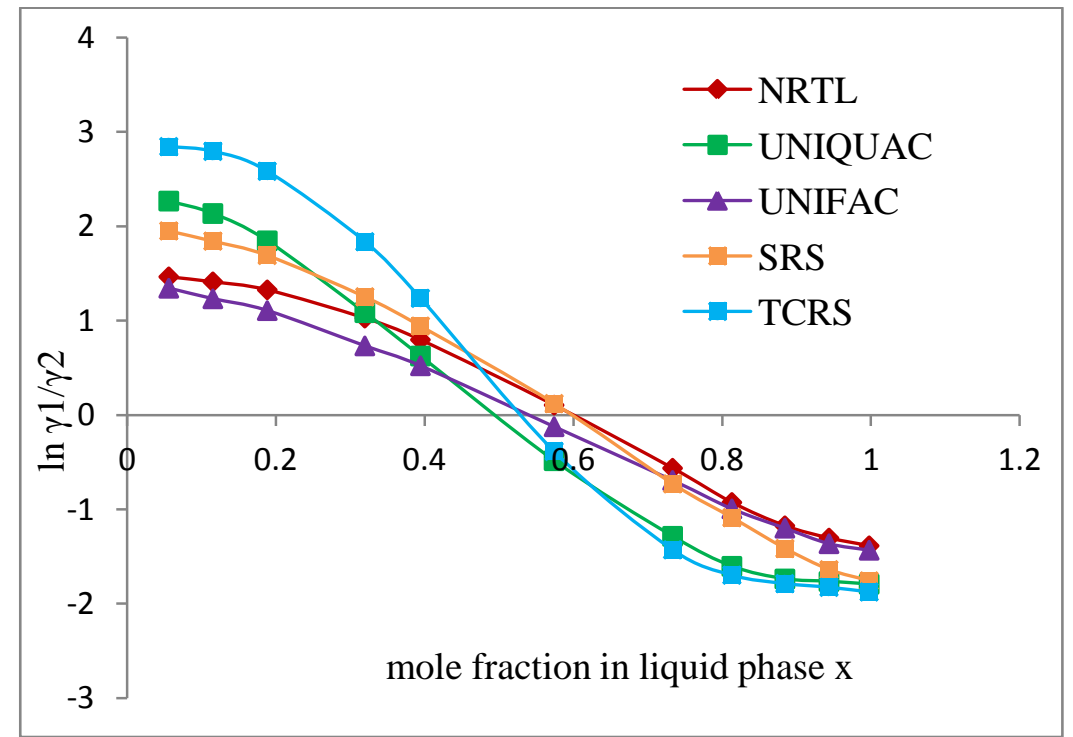

Fig. 8: Thermodynamic Consistency test of Acetone-methanol System Using Redlich Kiester method for NRTL, UNIQUAC, UNIFAC, SRS and TCRS models. 

Determination-Experimental and Theoretical studies. Journal of Applied Sciences Research., 14(1): 12-24.

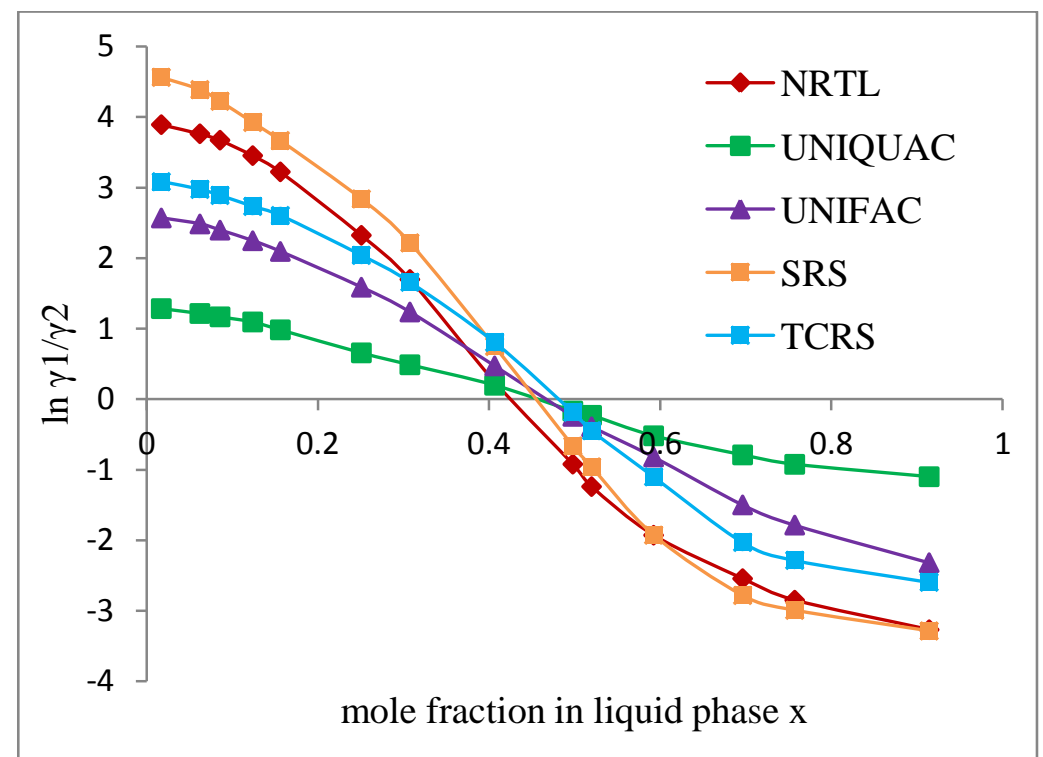

Fig. 9: Thermodynamic Consistency test of Ethanol-water System Using Redlich Kiester method for NRTL, UNIQUAC, UNIFAC, SRS and TCRS models.

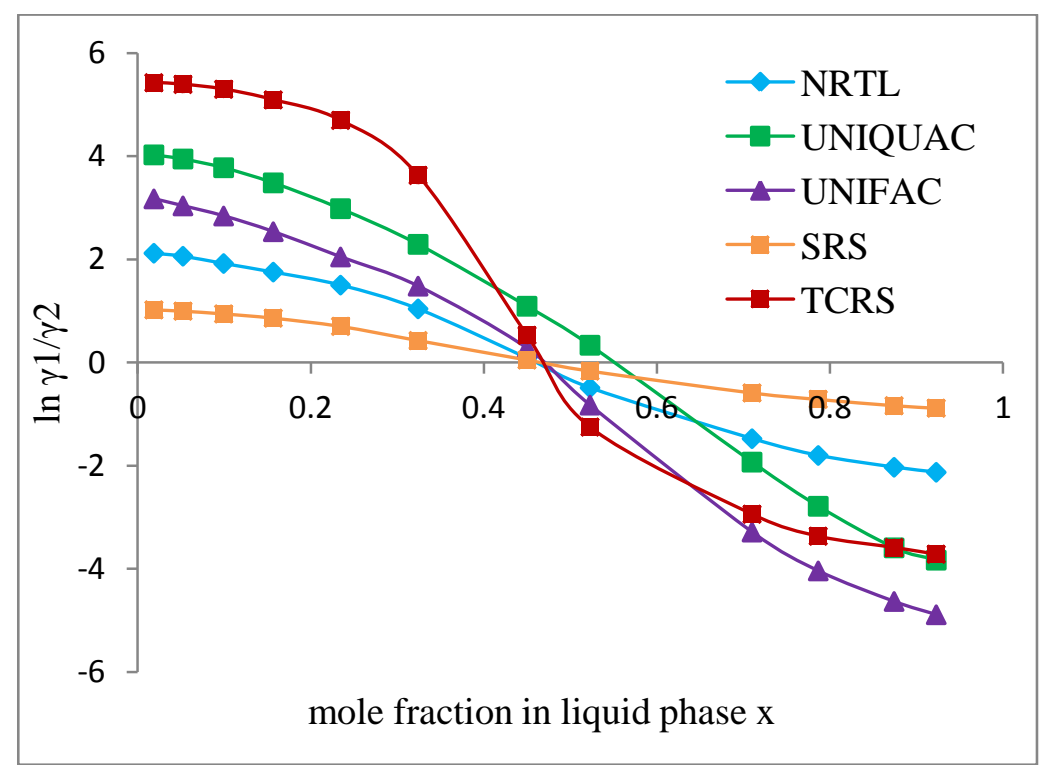

Fig. 10: Thermodynamic Consistency test of Ethanol-benzene System Using Redlich Kiester method for NRTL, UNIQUAC, UNIFAC, SRS and TCRS models.

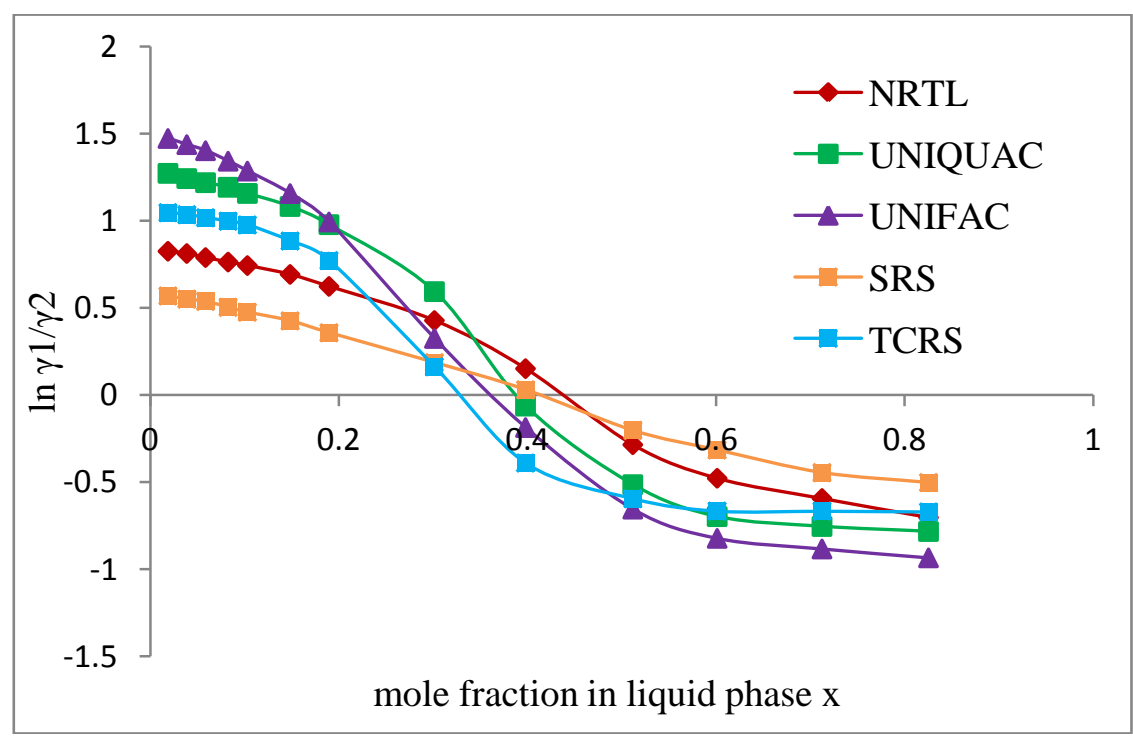

Fig. 11: Thermodynamic Consistency test of Methanol-water System Using Redlich Kiester method for NRTL, UNIQUAC, UNIFAC, SRS and TCRS models. These plots are made using activity coefficient from five models listed in tables 1-5.Area under the curve computed for these models are given in table 7. 
Citation: Manojkumar Selvakumar and Sivaprakash Baskaran, 2018. Advers Thermodynamic Analysis of Azeotropic mixtures for Vapour liquid Equilibrium Determination-Experimental and Theoretical studies. Journal of Applied Sciences Research., 14(1): 12-24.

Table 7: Thermodynamic Consistency test of five azeotropes system using RedlickKister method

\begin{tabular}{lllll}
\hline System & Area Under the Curve & & \\
\cline { 2 - 5 } & NRTL & UNIQUAC & UNIFAC & SRS \\
\hline Acetone-water & 0.0791 & 0.09075 & 0.01625 & 0.4445 \\
Acetone-methanol & 0.1789 & 0.08512 & 0.03621 & 0.00375 \\
Ethanol-water & 0.0158 & 0.0221 & 0.0201 & 0.2005 \\
Ethanol-benzene & 0.0165 & 0.4714 & 0.4041 & 0.1173 \\
Methanol-water & 0.0409 & 0.0052 & 0.0041 & 0.0054 \\
\hline
\end{tabular}

From the table it can be observed that value of TCRS model close to zero for acetone-water system. In a similar approach the acetone-methanol system shows good thermodynamic consistency for UNIFAC and UNIQUAC activity coefficient models whereas the ethanol-water own good concurrence with UNIFAC model. For ethanol-water system SRS and NRTL model gives better result of VLE consistency. Correspondingly UNIFAC and UNIQUAC models show appreciable thermodynamic consistency for methanol-water system.

\section{Conclusion:}

In case of VLE prediction of azeotropes, NRTL, UNIQUAC UNIFAC, SRS and TCRS models were tested for the systems namely Acetone-water, Acetonemethanol, Ethanol-water, Ethanol-benzene, and Methanol-water. The experimental VLE findings show that all the five systems show minimum boiling azeotropes (positive deviation from ideality). Major finding of the present work is the estimation of NRTL, UNIQUAC, SRS and TCRS parameters for the five systems. Parameter estimated in this work is not yet reported in any literature. These parameters can be utilized for VLE calculation at any pressure conditions. This paper will be beneficial for Chemical Industries distillation column design.

Future Work:

Future work is study on performance of entrainer for these five systems. The Entrainer selection is based on Relative volatility of the solvents. The experiment was carried out with the addition of entrainer, and the compositions of distillate and residue are found out at equilibrium temperatures. From this, the performance of the entrainer recovery is observed.

\section{ACKNOWLEDGEMENT}

Author would Author would like to express their gratitude to University grant commission of India (UGC) for financially supported this project under National fellow ship scheme (Project No: 17.1/2015-16/RGNF-2015-17-SC-TAM-24224).

Nomenclature:

$\mathrm{B}^{0} \& \mathrm{~B}^{1}=$ virial coefficients

$f_{\mathrm{i}} \quad=$ fugacity of the component $\mathrm{i}$ in standard state

$\mathrm{P} \quad=$ operating pressure

$\mathrm{P}_{\mathrm{i}}^{\text {Sat }}=$ saturation pressure of the component $\mathrm{i}$

$\mathrm{P}_{\mathrm{r}} \quad=$ reduced pressure

$\mathrm{q}_{\mathrm{i}} \quad$ = group volume parameter of the component $\mathrm{i}$

$\mathrm{Qi}=$ surface area parameter of the component $\mathrm{i}$

$\mathrm{r}_{\mathrm{i}} \quad=$ group volume parameter of the component $\mathrm{i}$

$\mathrm{R}_{\mathrm{i}} \quad$ = volume parameter of the component $\mathrm{i}$

$\mathrm{R}_{\mathrm{k}} \& \mathrm{Q}_{\mathrm{k}}=$ group area parameters

$\mathrm{T}_{\mathrm{r}} \quad=$ reduced temperature

$\mathrm{u}_{\mathrm{ij}} \quad=$ average interaction energy for the interaction of molecules of components $\mathrm{i}$ with the molecules of component $\mathrm{j}$

$\mathrm{x}_{\mathrm{i}} \quad=$ mole fraction in liquid phase of the component $\mathrm{i}$

$\mathrm{y}_{\mathrm{i}} \quad=$ mole fraction in vapour phase of the component $\mathrm{i}$

$\mathrm{z} \quad=$ coordination number which is usually taken 10

$\gamma_{\mathrm{i}} \quad=$ activity coefficient of the component $\mathrm{i}$

$\phi_{\mathrm{i}} \quad=$ fugacity coefficient of the components $i$

$\omega=$ accentric factor

$\phi_{\mathrm{i}}=$ segment or volume fraction of the component $\mathrm{i}$

$\theta_{\mathrm{i}} \quad=$ area fraction of the component $\mathrm{i}$

$\tau_{\mathrm{ji}}=$ adjustable parameters of the components $\mathrm{i}$ and $\mathrm{j}$

$\gamma_{i}{ }^{\mathrm{C}}=$ combinatorial term of the component $\mathrm{i}$

$\gamma_{i}{ }^{R}=$ residual term of the component $i$

\section{REFERENCES}

Seader, J.D., J. Henley, 2006. Separation process Principles, John Wiley and Sons Publication, New Jersey.

Geankoplis, C.J., 2003. Transport Process and Separation Process, Prentice Hall Publication, New Jersey.

Vivek Julka, Madhura Chiplunkar, L.O. Young, 2009. Selection Entariner for Azeotropic Distillation, Chemical Engineering Progress, pp: 47-53. Pahang.

Mohamad Azamudin, I., 2010. Effects of Temperature On Vapor Liquid Equilibrium Of Mtbe-Methanol Mixtures, Bachelor thesis, University Malaysia

Laszlo, H., 2013. Improvement of Batch Distillation Separation of Azeotropic Mixtures, Phd thesis, Budapest University of Technology and Economics.

Gadekar, S.V., R.V. Naik, J.D. Bapat, 2004. Acetic Acid-Water-Toluene System Batch Distillation Parameters for Heterogeneous Azeotropic systems, Chemical Engineering World, 44.

Narayanan, K.V., 2004. A Textbook of Chemical Engineering Thermodynamics, Prentice Hall India Learning Private Limited.

Hilmen, E.K., 2000. Separation of Azeotropic Mixtures Tools for Analysis and Studies on Batch Distillation Operation, Phd thesis, Norwegian University of Science and Technology.

Swietoslawski, W., 1963. Azeotropy and Polyazeotropy, Oxford Pergamon Press, London.

Managobinda, B., 2010. VLE modeling Using Unifac Group Contribution Method and its application in Distillation Column Design and Steady State Simulation, Dissertation for Bachelor thesis, National Institute of Technology, Rourkela.

Ngema, P.T., 2010. Separation process for high purity ethanol production, Dissertation for the M.S. Degree, Durban University of Technology.

Kannan, A., 2003. Short Term Training Programme on Modelling, Simulation and Analysis of Enhanced Distillation Process, Indian institute of technology.

Rao, Y.V.C., 1997. Chemical Engineering Thermodynamics, Universities Press India Limited, Hyderabad.

Renon, H., J.M. Prausnitz, 1968. Local Compositions in Thermodynamic Excess Functions for Liquid Mixtures, Journal of American Institute of Chemical Engineers, 14: 135-144. 
Anderson, T.F., J.M Prausnitz, 1978. Application of the UNIQUAC equation to calculation of multicomponent phase equilibria, Industrial and Engineering Chemistry Process Design and Development, 17(4): 561-567.

Fredenslund, A., J. Gmehling, M.L. Michelson, P. Rasmussen, 1977. Computerized Design of Multicomponent Distillation Columns using the UNIFAC Group Contribution Method, Industrial and Engineering Chemistry Process Design and Development, 16(4): 450-462.

Fredenslund, A., R.L. Jones, J.M. Prausnitz, 1975. Group-Contribution Estimation of Activity Coefficients in Nonideal Liquid Mixtures, American Institute of Chemical Engineering, 21(6): 1086-1099.

Sabarathinam, P.L., B. Sivaprakash, 2002. Theoretically consistent modified Local composition and Flory-huggins equations in VLE data prediction, Mtech Thesis, Annamalai University.

Philip Jackson, L., A. Richard Wilsak, 1995. Thermodynamic consistency tests based on the Gibbs-Duhem equation applied to isothermal, binary vapourliquid equilibrium data evaluation and model testing, Fluid Phase Equilibria, 103(2): 155-197.

Redlich, O., A.T. Kister, 1948. Algebraic Representation of Thermodynamic Properties and the Classification of Solutions, Industrial and Engineering Chemistry, 40(2): 345-348.

Luben, W.L., L.A. Wenzel, 1988. Chemical process analysis, Prentice-Hall International Series, New Jersey. 